\title{
The Cos-Poisson model with a novel count regression analysis
}

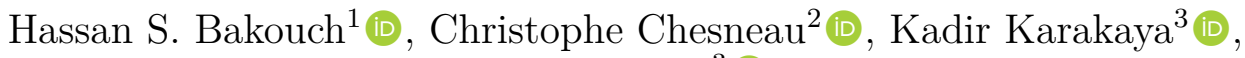 \\ Coşkun $\mathrm{Kus}^{* 3}$ (1) \\ ${ }^{1}$ Department of Mathematics, Faculty of Science, Tanta University, Tanta, Egypt \\ ${ }^{2}$ Université de Caen, LMNO, Campus II, Science 3, 14032, Caen, France \\ ${ }^{3}$ Department of Statistics, Faculty of Science, Selcuk University, 42250, Konya, Turkey
}

\begin{abstract}
In this paper, we propose a new generalization of the Poisson distribution by using the concept of the weighted distribution; a trigonometric weight with the cosine function is used. We derive some distributional properties of the new distribution, such as the cumulative distribution function, moment generating function, factorial moments, and index of dispersion. Then, the related model is considered for modeling purposes, with estimation of the model parameters performed via several methods. Zero-inflated count regression analysis is introduced by using the new distribution. Finally, we provide two applications of the obtained results on practical data sets.
\end{abstract}

Mathematics Subject Classification (2020). 60E05, 62E15, 62F10

Keywords. confidence interval, count data, count regression, index of dispersion, zero-inflated data

\section{Introduction}

Many areas of applied sciences require precise models for processing count data (such as ecology, environmental science, insurance, etc.). To obtain such distributions, there is a need for new discrete distributions with desirable properties. Among the simple techniques in this regard, there are the weighted distributions introduced by Rao [15]. These techniques aim to create flexible distributions by the use of a tuning weight function and a well-established (simple) baseline distribution. For further details, we may refer the reader to Patil and Rao $[13,14]$ for the general formalism with discussions, Castillo and Casany [6] for the Poisson distribution as a baseline, Bhati and Joshi [4] for the geometric distribution as a baseline and Bakouch [3] for the negative binomial Lindley distribution as a baseline, and the references therein. The mathematical backgrounds of the discrete weighted distributions can be formulated as follows. For a random variable $X$ following a discrete distribution defined by a probability mass function (pmf) denoted by $f_{*}(x)$ and

\footnotetext{
*Corresponding Author.

Email addresses: hassan.bakouch@science.tanta.edu.eg (H.S. Bakouch), christophe.chesneau@gmail.com (C. Chesneau), kkarakaya@selcuk.edu.tr (K. Karakaya), coskun@selcuk.edu.tr (C. Kuş)

Received: 21.05.2020; Accepted: 27.11.2020
} 
a weight function denoted by $w(x)$, the corresponding weighted distribution is defined by the following pmf:

$$
f(x)=\frac{1}{E[w(X)]} w(x) f_{*}(x),
$$

where $E[w(X)]$ denotes the expectation of the random variable $w(X)$. The most used kind of weight function is the polynomial one, i.e., $w(x)=x, w(x)=w(x ; r)=x^{r}$ or $w(x)=w(x ; a, r)=(x+a)^{r}$, with success in count data modeling. On this topic, we again refer to Castillo and Casany [6] for the Poisson distribution and Bhati and Joshi [4] for the geometric distribution.

In this paper, we introduce a new generalization of the Poisson distribution with the use of a cosine weight function defined by $w(x)=w(x ; \beta)=[\cos (\beta x)]^{2}$, called the cosine Poisson (CosPois) distribution. We thus follow the spirit of the cosine geometric distribution introduced by Chesneau et al. [7], but with the Poisson distribution as a baseline. The motivations behind this choice are as follows. Thanks to the oscillating nature of the cosine function, we show that the desirable properties of the Poisson distribution are significantly enriched. The CosPois distribution can be over-dispersed or under-dispersed, unlike Poisson distribution. It can be more flexible than the Poisson distribution for modeling practical data through this property. It is also observed that the CosPois distribution is better for modeling the number of outbreaks of strikes (under-dispersed data) in UK coal mining industries, as shall be seen later.

Regarding inference on the model parameters, we consider three methods such as maximum likelihood, proportion and moment estimators. Furthermore, two types of confidence intervals (CIs) of parameters are discussed through asymptotic distributions of maximum likelihood estimators and likelihood ratio statistic. The simulation study revealed that the proportion type estimates are competitor to the others in terms of the mean square error (MSE) criterion. The robustness of the proportion type estimates is detected. It is also observed that the CIs based on likelihood ratio statistic behave like the MLE based CIs in terms of the coverage probabilities through another simulation study.

Also, the CosPois distribution is adapted to a count regression analysis with the zeroinflation procedure and it is applied to a practical data. It is demonstrated that the zeroinflated CosPois count regression analysis can be an alternative to well-known zero-inflated count regressions, such as Poisson and negative binomial via the Akaike's Information Criterion (AIC).

The rest of the article is summarized as follows. Section 2 presents the CosPois distribution, with expressions of the corresponding pmf, cumulative distribution and hazard rate functions. Some mathematical properties complete this presentation in Section 3. The data generation algorithm for the CosPois distribution is provided in Section 4. In Section 5, five different estimators are studied and the estimation of the model parameters with a simulation study is performed. Two interval estimation methods are discussed for the parameters of the CosPois distribution in Section 6. A practical data analysis is also presented in Section 7. In Section 8, a zero-inflated count regression analysis is introduced and an illustrative example with practical data is presented to demonstrate the applicability of the introduced regression analysis. The concluding remarks are provided in Section 9 .

\section{The CosPois distribution}

Hereafter, let $T$ be a random variable following the Poisson distribution with parameter $\lambda>0$, i.e., with pmf: $f_{*}(x)=f_{*}(x ; \lambda)=e^{-\lambda} \lambda^{x} / x !, x \in \mathbb{N}$ and cumulative distribution function (cdf) denoted by $F_{*}(x ; \lambda)=P(T \leq x), x \in \mathbb{R}$. Let $w(x)=w(x ; \beta)=[\cos (\beta x)]^{2}$. Then, we define the CosPois distribution with parameters $\beta \in(0, \pi / 2)$ and $\lambda>0$ by the pmf given by 


$$
f(x ; \boldsymbol{\theta})=C_{\boldsymbol{\theta}}[\cos (\beta x)]^{2} \frac{\lambda^{x}}{x !}, \quad x \in \mathbb{N},
$$

where

$$
\begin{aligned}
C_{\boldsymbol{\theta}} & =e^{-\lambda}\{E[w(T ; \beta)]\}^{-1} \\
& =2 e^{-\lambda}\left\{1+e^{-\lambda[1-\cos (2 \beta)]} \cos [\lambda \sin (2 \beta)]\right\}^{-1},
\end{aligned}
$$

and $\boldsymbol{\theta}=(\beta, \lambda)$.

The value of $C_{\boldsymbol{\theta}}$ follows from Lemma 3.1 formulated later (with $m=0, t=\beta$ and $\kappa=\lambda$ ). By taking $\beta=0$, the CosPois distribution is reduced to the former Poisson distribution with parameter $\lambda$. The distribution with $\operatorname{pmf}(2.1)$ is denoted by $\operatorname{CosPois}(\boldsymbol{\theta})$ for abbreviating. It is noticed here that the $\operatorname{pmf}(2.1)$ is valid for the case $\beta>\pi / 2$ but it exhibits the same pmf periodically. That is, $\operatorname{CosPois}(\boldsymbol{\theta})=\operatorname{CosPois}((k+1) \pi-\beta, \lambda)$, for $\beta \in(0, \pi / 2)$ and $k=0,1, \ldots$

Furthermore, we have

$$
\frac{f(x+1 ; \boldsymbol{\theta})}{f(x ; \boldsymbol{\theta})}=\frac{\lambda}{x+1} \frac{\{\cos [\beta(x+1)]\}^{2}}{[\cos (\beta x)]^{2}} .
$$

Clearly, without restriction on $\beta$, this ratio is a non-monotonic function with respect to $x$, implying the possible multimodality of the CosPois distribution. Some plots of the pmf of the CosPois distribution are given in Figure 1 for selected values of parameters. From Figure 1, it is observed that the pmf can be unimodal or periodically increasing and decreasing. This implies that the CosPois distribution potentially becomes a candidate for modeling the applications on count data.
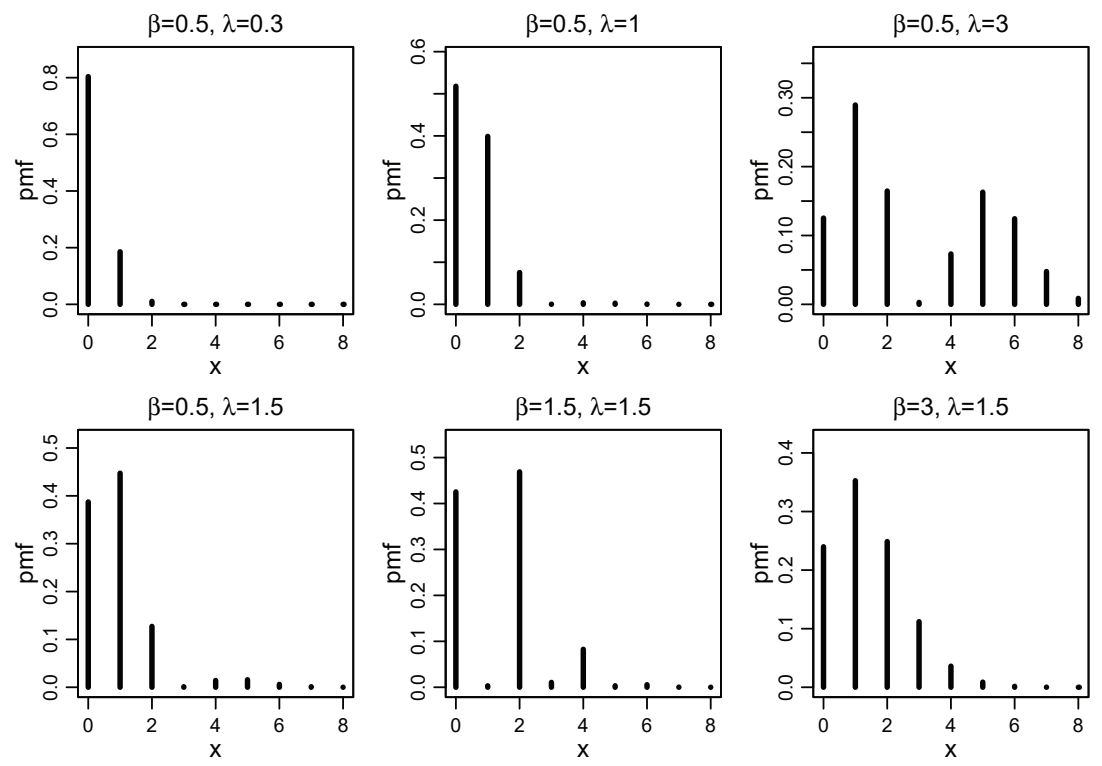

Figure 1. Pmf plot for selected parameter values

Also, by using the following formula: $[\cos (a)]^{2}=(1 / 2)[1+\cos (2 a)], a \in \mathbb{R}$, the cdf of the CosPois distribution can be expressed as

$$
F(t ; \boldsymbol{\theta})=C_{\boldsymbol{\theta}}^{*}\left[F_{*}(t ; \lambda)+\Upsilon(t ; \boldsymbol{\theta})\right], \quad t \in \mathbb{R},
$$

where

$$
C_{\boldsymbol{\theta}}^{*}=C_{\boldsymbol{\theta}} e^{\lambda} 2^{-1}=\left\{1+e^{-\lambda[1-\cos (2 \beta)]} \cos [\lambda \sin (2 \beta)]\right\}^{-1}
$$


and $\Upsilon(t ; \boldsymbol{\theta})=\operatorname{Re}\left[F_{*}\left(t ; \lambda e^{2 i \beta}\right)\right]$. After some algebraic manipulations, we can show that

$$
\Upsilon(t ; \boldsymbol{\theta})=e^{-\lambda} \sum_{x=0}^{[t]} \frac{\lambda^{x}}{x !}[\cos (2 \beta)]^{x} \sum_{k=0}^{[x / 2]}\left(\begin{array}{c}
x \\
2 k
\end{array}\right)(-1)^{k}[\tan (2 \beta)]^{2 k},
$$

where $[t]$ denotes the integer just less than or equal to $t$.

\section{Mathematical properties}

We first consider the following useful lemma.

Lemma 3.1. For any $\kappa, m, t \in \mathbb{R}$, we have

$$
\sum_{x=0}^{+\infty}\{\cos [t(x+m)]\}^{2} \frac{\kappa^{x}}{x !}=\frac{1}{2} e^{\kappa}\left\{1+e^{-\kappa[1-\cos (2 t)]} \cos [\kappa \sin (2 t)+2 m t]\right\} .
$$

Proof. First of all, by using the following formula: $[\cos (a)]^{2}=(1 / 2)[1+\cos (2 a)], a \in \mathbb{R}$, and the series expansion of the exponential function, we get

$$
\sum_{x=0}^{+\infty}\{\cos [t(x+m)]\}^{2} \frac{\kappa^{x}}{x !}=\frac{1}{2} e^{\kappa}\left(1+e^{-\kappa} \sum_{x=0}^{+\infty} \cos [2 t(x+m)] \frac{\kappa^{x}}{x !}\right) .
$$

Now, remark that

$$
\begin{aligned}
& \sum_{x=0}^{+\infty} \cos [2 t(x+m)] \frac{\kappa^{x}}{x !}=\operatorname{Re}\left[e^{i m 2 t} \sum_{x=0}^{+\infty} \frac{\left(\kappa e^{i 2 t}\right)^{x}}{x !}\right]=\operatorname{Re}\left[e^{i m 2 t} e^{\kappa e^{i 2 t}}\right] \\
& =e^{\kappa \cos (2 t)} \operatorname{Re}\left\{e^{i[\kappa \sin (2 t)+m 2 t]}\right\} \\
& =e^{\kappa \cos (2 t)} \operatorname{Re}\{\cos [\kappa \sin (2 t)+m 2 t]+i \cos [\kappa \sin (2 t)+m 2 t]\} \\
& =e^{\kappa \cos (2 t)} \cos [\kappa \sin (2 t)+m 2 t] .
\end{aligned}
$$

The desired equality follows by combining the equalities above, ending the proof of Lemma 3.1 .

Hereafter, let $X$ be a random variable following the CosPois distribution with parameters $\beta \geq 0$ and $\lambda>0$.

The following proposition presents the probability generating function (pgf) of $X$.

Proposition 3.2. The probability generating function of $X$ is given by

$$
G_{X}(s)=E\left(s^{X}\right)=e^{-\lambda(1-s)} \frac{1+e^{-s \lambda[1-\cos (2 \beta)]} \cos [s \lambda \sin (2 \beta)]}{1+e^{-\lambda[1-\cos (2 \beta)]} \cos [\lambda \sin (2 \beta)]}, \quad s \in \mathbb{R} .
$$

Proof. It follows from Lemma 3.1 with $m=0, t=\beta$ and $\kappa=s \lambda \in \mathbb{R}$ that

$$
\begin{aligned}
G_{X}(s) & =C_{\boldsymbol{\theta}} \sum_{x=0}^{+\infty}[\cos (\beta x)]^{2} \frac{(s \lambda)^{x}}{x !} \\
& =C_{\boldsymbol{\theta}} \frac{1}{2} e^{s \lambda}\left\{1+e^{-s \lambda[1-\cos (2 \beta)]} \cos [s \lambda \sin (2 \beta)]\right\} \\
& =e^{-\lambda(1-s)} \frac{1+e^{-s \lambda[1-\cos (2 \beta)]} \cos [s \lambda \sin (2 \beta)]}{1+e^{-\lambda[1-\cos (2 \beta)]} \cos [\lambda \sin (2 \beta)]} .
\end{aligned}
$$

This ends the proof of Proposition 3.2.

Remark 3.3. It follows from Proposition 3.2 that

$$
G_{X}(s)=G_{T}(s) \frac{1+e^{-s \lambda[1-\cos (2 \beta)]} \cos [s \lambda \sin (2 \beta)]}{1+e^{-\lambda[1-\cos (2 \beta)]} \cos [\lambda \sin (2 \beta)]}, \quad s \in \mathbb{R},
$$

where $G_{T}(s)$ denotes the pgf of $T$. In this sense, $G_{X}(s)$ is a weighted version of $G_{T}(s)$. 
We deduce the moment generating function of $X$ :

$$
M_{X}(t)=E\left(e^{t X}\right)=e^{-\lambda\left(1-e^{t}\right)} \frac{1+e^{-e^{t} \lambda[1-\cos (2 \beta)]} \cos \left[e^{t} \lambda \sin (2 \beta)\right]}{1+e^{-\lambda[1-\cos (2 \beta)]} \cos [\lambda \sin (2 \beta)]} .
$$

Also, the characteristic function of $X$ is given by

$$
\varphi_{X}(t)=E\left(e^{i t X}\right)=e^{-\lambda\left(1-e^{i t}\right)} \frac{1+e^{-e^{i t} \lambda[1-\cos (2 \beta)]} \cos \left[e^{i t} \lambda \sin (2 \beta)\right]}{1+e^{-\lambda[1-\cos (2 \beta)]} \cos [\lambda \sin (2 \beta)]} .
$$

The factorial moments of $X$ are investigated in the following result.

Proposition 3.4. The $r^{\text {th }}$ factorial moment of $X$ is given by

$$
\begin{aligned}
\mu_{(r), X}^{\prime} & =E[X(X-1) \cdots(X-r+1)] \\
& =\lambda^{r} \frac{1+e^{-\lambda[1-\cos (2 \beta)]} \cos [\lambda \sin (2 \beta)+2 r \beta]}{1+e^{-\lambda[1-\cos (2 \beta)]} \cos [\lambda \sin (2 \beta)]} .
\end{aligned}
$$

Proof. It follows from some algebraic manipulations (mainly changes of indices) and Lemma 3.1 with $m=r, t=\beta$ and $\kappa=\lambda$ that

$$
\begin{aligned}
\mu_{(r), X}^{\prime} & =C_{\boldsymbol{\theta}} \sum_{x=r}^{+\infty} x(x-1) \cdots(x-r+1)[\cos (\beta x)]^{2} \frac{\lambda^{x}}{x !} \\
& =C_{\boldsymbol{\theta}} \sum_{x=r}^{+\infty}[\cos (\beta x)]^{2} \frac{\lambda^{x}}{(x-r) !}=C_{\beta, \lambda} \lambda^{r} \sum_{x=0}^{+\infty}\{\cos [\beta(x+r)]\}^{2} \frac{\lambda^{x}}{x !} \\
& =C_{\boldsymbol{\theta}} \lambda^{r} e^{\lambda} \frac{1}{2}\left\{1+e^{-\lambda[1-\cos (2 \beta)]} \cos [\lambda \sin (2 \beta)+2 r \beta]\right\} \\
& =\lambda^{r} \frac{1+e^{-\lambda[1-\cos (2 \beta)]} \cos [\lambda \sin (2 \beta)+2 r \beta]}{1+e^{-\lambda[1-\cos (2 \beta)]} \cos [\lambda \sin (2 \beta)]} .
\end{aligned}
$$

This ends the proof of Proposition 3.4.

Remark 3.5. It follows from Proposition 3.4 that

$$
\mu_{(r), X}^{\prime}=\mu_{(r), T}^{\prime} \frac{1+e^{-\lambda[1-\cos (2 \beta)]} \cos [\lambda \sin (2 \beta)+2 r \beta]}{1+e^{-\lambda[1-\cos (2 \beta)]} \cos [\lambda \sin (2 \beta)]},
$$

where $\mu_{(r), T}^{\prime}$ denotes the $r^{t h}$ factorial moment of $T$. In this sense, $\mu_{(r), X}^{\prime}$ is a weighted version of $\mu_{(r), T}^{\prime}$.

Some important consequences of Proposition 3.4 are presented below. The mean of $X$ is given by

$$
\mu=E(X)=\mu_{(1)}^{\prime}=\lambda \frac{1+e^{-\lambda[1-\cos (2 \beta)]} \cos [\lambda \sin (2 \beta)+2 \beta]}{1+e^{-\lambda[1-\cos (2 \beta)]} \cos [\lambda \sin (2 \beta)]} .
$$

The other raw moments of $X$ can be deduced from the factorial moments, the same for the central moments.

In particular, the variance of $X$ can be obtained by

$$
\begin{aligned}
\sigma^{2} & =\operatorname{Var}(X)=E\left[(X-\mu)^{2}\right]=\mu_{(2)}^{\prime}+\mu-\mu^{2} \\
& =\lambda^{2} \frac{1+e^{-\lambda[1-\cos (2 \beta)]} \cos [\lambda \sin (2 \beta)+4 \beta]}{1+e^{-\lambda[1-\cos (2 \beta)]} \cos [\lambda \sin (2 \beta)]}+\lambda \frac{1+e^{-\lambda[1-\cos (2 \beta)]} \cos [\lambda \sin (2 \beta)+2 \beta]}{1+e^{-\lambda[1-\cos (2 \beta)]} \cos [\lambda \sin (2 \beta)]} \\
& -\left(\lambda \frac{1+e^{-\lambda[1-\cos (2 \beta)]} \cos [\lambda \sin (2 \beta)+2 \beta]}{1+e^{-\lambda[1-\cos (2 \beta)]} \cos [\lambda \sin (2 \beta)]}\right)^{2}
\end{aligned}
$$


The dispersion index is defined by

$$
\begin{aligned}
& I(X)=\frac{\sigma^{2}}{\mu}=1+ \\
& \lambda\left[\frac{1+e^{-\lambda[1-\cos (2 \beta)]} \cos [\lambda \sin (2 \beta)+4 \beta]}{1+e^{-\lambda[1-\cos (2 \beta)]} \cos [\lambda \sin (2 \beta)+2 \beta]}-\frac{1+e^{-\lambda[1-\cos (2 \beta)]} \cos [\lambda \sin (2 \beta)+2 \beta]}{1+e^{-\lambda[1-\cos (2 \beta)]} \cos [\lambda \sin (2 \beta)]}\right] .
\end{aligned}
$$

The sign of the bracket term is determinant to evaluate the dispersed nature of the CosPois distribution; we can have $I(X)<1, I(X)=1$ (for $\beta=0$ ) and $I(X)>1$, depending on the values of $\beta$ and $\lambda$. This versatility is illustrated by Figure 2 .

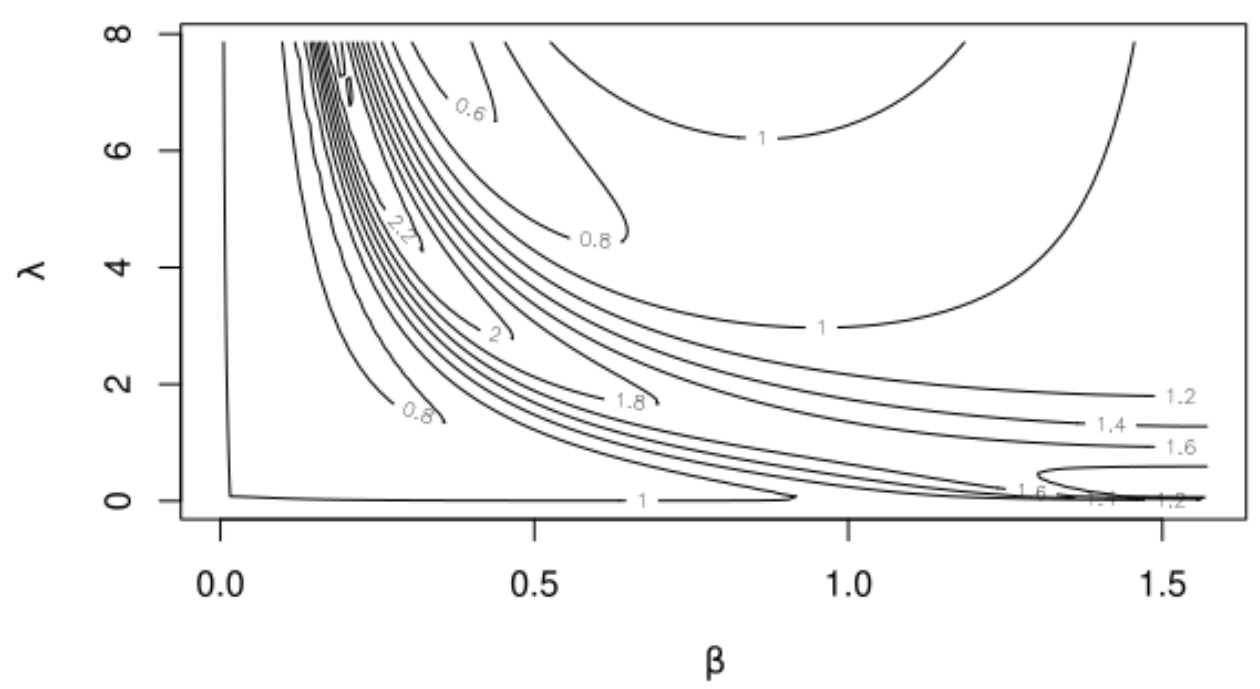

Figure 2. Contour plot for dispersion index

From Figure 2, it is observed that the dispersion index of the CosPois distribution can be over-dispersed, under-dispersed or non-dispersed. Furthermore, we have

$$
\underset{\beta \in\left(0, \frac{\pi}{2}\right), \lambda>0}{\arg \min } I(X)=(0.2236301,12.8414043), \underset{\beta \in\left(0, \frac{\pi}{2}\right), \lambda>0}{\arg \max } I(X)=(0.01221437,127.93516246)
$$

and

$$
\min _{\beta \in\left(0, \frac{\pi}{2}\right), \lambda>0} I(X)=0.4296437, \max _{\beta \in\left(0, \frac{\pi}{2}\right), \lambda>0} I(X)=2.959501 .
$$

So the dispersion index lies between $(0.4296437,2.959501)$ for the CosPois distribution. The final note on the dispersion index is that

$$
\lim _{\beta \rightarrow 0} I(X)=1 \text { and } \lim _{\beta \rightarrow \frac{\pi}{2}, \lambda \rightarrow \infty} I(X)=1 .
$$

The range for the dispersion index is given above for the CosPois distribution and we observe that it could be 1 (non-dispersed), less (under-dispersed) or greater than 1 (overdispersed). This indicates that the new distribution can be treated as a distribution of a response variable in a count regression analysis. It can also be concluded that a count regression constructed with the introduced distribution theory can be used to model all types of response data, such as non-dispersed, over-dispersed and under-dispersed. 


\section{Data generation algorithm}

In this section, an algorithm is proposed to generate the data from the CosPois distribution with pmf as given in (2.1). It is given as follows:

Step 1. $i=0$,

Step 2. Generate a datum from the standard uniform distribution, say $u$,

Step 3. $i=i+1$,

Step 4. If $u>\sum_{j=0}^{i-1} f(j ; \boldsymbol{\theta})$ goto Step 3 else goto Step 5 ,

Step 5. $x=i-1$.

This algorithm suggests that $x$ is a realization of the random variable $X$ which follows from the CosPois distribution with pmf (2.1). In the data generating algorithm, one can use the recurrence relation $(2.3)$ to obtain the $f(j ; \boldsymbol{\theta})$ in Step 4 . Then, one simply has to calculate the probability of zero value explicitly and then use the recursive formula to determine all other probabilities without evaluating the $x$ !.

\section{Point estimators for unknown distribution parameters}

In this section, the maximum likelihood, proportion types and moment estimators are described for the parameter $\boldsymbol{\theta}$ of the CosPois distribution. The bias and MSEs of these estimators are also investigated through a simulation study.

\subsection{Method of maximum likelihood}

We can estimate the CosPois model parameters, i.e., $\beta$ and $\lambda$, by the maximum likelihood method. Let $x_{1}, \ldots, x_{n}$ be $n$ independent realizations of a random variable $X$ following the CosPois distribution with parameters $\beta$ and $\lambda$. Then, by use of the pmf given by (2.1), the log-likelihood function is defined by

$$
\begin{aligned}
\ell_{n}(\boldsymbol{\theta}) & =\log \left[\prod_{i=1}^{n} f\left(x_{i} ; \boldsymbol{\theta}\right)\right] \\
& =n \log \left[C_{\beta, \lambda}\right]+2 \sum_{i=1}^{n} \log \left[\cos \left(\beta x_{i}\right)\right]+\log (\lambda) \sum_{i=1}^{n} x_{i}-\sum_{i=1}^{n} \log \left(x_{i} !\right),
\end{aligned}
$$

where $\boldsymbol{\theta}=(\beta, \lambda)$. Then, the maximum likelihood estimate (MLE) $\widehat{\boldsymbol{\theta}}=(\widehat{\beta}, \widehat{\lambda})$ for $\boldsymbol{\theta}$ is defined by

$$
\widehat{\boldsymbol{\theta}}=\underset{(\beta, \lambda) \in\left[0, \frac{\pi}{2}\right] \times(0,+\infty)}{\arg \max } \ell_{n}(\boldsymbol{\theta}) .
$$

Then, $\widehat{\boldsymbol{\theta}}$ is the solution of the following non-linear equations: $\partial \ell_{n}(\boldsymbol{\theta}) / \partial \beta=0$ and $\partial \ell_{n}(\boldsymbol{\theta}) / \partial \lambda=$ 0 , with

$$
\frac{\partial \ell_{n}(\boldsymbol{\theta})}{\partial \beta}=n \frac{\partial C_{\beta, \lambda} / \partial \beta}{C_{\beta, \lambda}}-2 \sum_{i=1}^{n} x_{i} \frac{\sin \left(\beta x_{i}\right)}{\cos \left(\beta x_{i}\right)}
$$

and

$$
\frac{\partial \ell_{n}(\boldsymbol{\theta})}{\partial \lambda}=n \frac{\partial C_{\boldsymbol{\theta}} / \partial \lambda}{C_{\beta, \lambda}}+\frac{1}{\lambda} \sum_{i=1}^{n} x_{i}
$$

where, by using (2.2),

$$
\frac{\partial C_{\boldsymbol{\theta}}}{\partial \beta}=\frac{4 \lambda \sin [2 \beta+\lambda \sin (2 \beta)] e^{-\lambda \cos (2 \beta)}}{\left\{e^{2 \lambda[\sin (\beta)]^{2}}+\cos [\lambda \sin (2 \beta)]\right\}^{2}}
$$


and

$$
\frac{\partial C_{\boldsymbol{\theta}}}{\partial \lambda}=-\frac{2 e^{-\lambda \cos (2 \beta)}\left(e^{2 \lambda[\sin (\beta)]^{2}}+\cos [2 \beta+\lambda \sin (2 \beta)]\right)}{\left\{e^{2 \lambda[\sin (\beta)]^{2}}+\cos [\lambda \sin (2 \beta)]\right\}^{2}} .
$$

The MLEs $\widehat{\beta}$ and $\hat{\lambda}$ have no closed forms. However, we use numerical methods to determine them with a high level of numerical precision.

\subsection{Method of proportions}

Khan [10] proposed the proportions estimators (PEs) to estimate the unknown parameters of discrete Weibull distribution. The same idea is also used in Akdoğan et al. [1] and Kuş et al. [11] and can be applied to estimate the CosPois parameters. Let $X_{1}, X_{2}, \ldots, X_{n}$ be a random sample from the $\operatorname{CosPois}(\boldsymbol{\theta})$ distribution. Furthermore, let $x_{1}, x_{2}, \ldots, x_{m}(m \leq n)$ be the realization of this sample with frequencies $b_{1}, b_{2}, \ldots, b_{m}$, respectively. Then, proportion type estimators can be defined by

$$
\begin{aligned}
& \widehat{\boldsymbol{\theta}}_{2}=\underset{(\beta, \lambda) \in\left[0, \frac{\pi}{2}\right] \times(0,+\infty)}{\arg \min }\left(\sum_{i=1}^{m}\left(f\left(x_{i} ; \boldsymbol{\theta}\right)-\frac{b_{i}}{n}\right)^{2}\right), \\
& \widehat{\boldsymbol{\theta}}_{3}=\underset{(\beta, \lambda) \in\left[0, \frac{\pi}{2}\right] \times(0,+\infty)}{\arg \min }\left(\sum_{i=1}^{m}\left|f\left(x_{i} ; \boldsymbol{\theta}\right)-\frac{b_{i}}{n}\right|\right),
\end{aligned}
$$

and

$$
\widehat{\boldsymbol{\theta}}_{4}=\underset{(\beta, \lambda) \in\left[0, \frac{\pi}{2}\right] \times(0,+\infty)}{\arg \min }\left(\max \left(\left|f\left(x_{1} ; \boldsymbol{\theta}\right)-\frac{b_{1}}{n}\right|, \ldots,\left|f\left(x_{m} ; \boldsymbol{\theta}\right)-\frac{b_{m}}{n}\right|\right)\right),
$$

where $f(x ; \boldsymbol{\theta})$ is the CosPois pmf given in (2.1). The PEs given in (5.3)-(5.5) can be obtained by optim function in $\mathrm{R}$ with L-BFGS-B algorithm.

\subsection{Method of moments}

In this subsection, the moments estimates (MEs) are obtained based on a sample $X_{1}, X_{2}, \ldots, X_{n}$ from the CosPois distribution with realization $x_{1}, x_{2}, \ldots, x_{m}$. Using (3.1), the MEs of the parameter $\boldsymbol{\theta}$ are obtained by solving the following simultaneous equations:

$$
\frac{\lambda\{1+\exp (-\lambda(1-\cos (2 \beta))) \cos (\lambda \sin (2 \beta)+2 \beta)\}}{1+\exp (-\lambda(1-\cos (2 \beta))) \cos (\lambda \sin (2 \beta))}-\frac{1}{n} \sum_{i=1}^{n} x_{i}=0
$$

and

$$
\begin{aligned}
& \frac{\lambda^{2}\{1+\exp (-\lambda(1-\cos (2 \beta))) \cos (\lambda \sin (2 \beta)+4 \beta)\}}{1+\exp (-\lambda(1-\cos (2 \beta))) \cos (\lambda \sin (2 \beta))} \\
& +\frac{\lambda\{1+\exp (-\lambda(1-\cos (2 \beta))) \cos (\lambda \sin (2 \beta)+2 \beta)\}}{1+\exp (-\lambda(1-\cos (2 \beta))) \cos (\lambda \sin (2 \beta))}-\frac{1}{n} \sum_{i=1}^{n} x_{i}^{2}=0 .
\end{aligned}
$$

Equations (5.6) and (5.7) can be solved numerically. The MEs can also be defined by

$$
\widehat{\boldsymbol{\theta}}_{5}=\underset{(\beta, \lambda) \in\left[0, \frac{\pi}{2}\right] \times(0,+\infty)}{\arg \min }\left(\max \left(\left|m_{1}(\boldsymbol{\theta})\right|,\left|m_{2}(\boldsymbol{\theta})\right|\right)\right),
$$

where $m_{1}(\boldsymbol{\theta})$ and $m_{2}(\boldsymbol{\theta})$ are left hand side of equations given in (5.6) and (5.7), respectively. The MEs given in (5.8) can be obtained by optim function in R with L-BFGS-B algorithm. 


\subsection{Simulation study for the point estimates}

In this subsection, the bias and MSEs of MLEs, PEs and MEs of parameters are estimated with 5000 trials. The algorithm described in Section 4 is used to generate the data from the $\operatorname{CosPois}(\boldsymbol{\theta})$ distribution. The L-BFGS-B method is applied to get the estimates given in (5.2)-(5.5) and (5.8) numerically. It is a quasi-Newton method which is available in $\mathrm{R}$ function optim.

The numerical results of the simulation study reported in Tables 1-2 present the bias and MSEs of estimates for the true parameter vectors $\boldsymbol{\theta}=(1.1,0.8),(0.9,0.7),(1.5,5)$ and $(0.4,5)$. These tables indicate that the bias and MSEs of MLEs, PEs and MEs are close to zero when $n$ increases as expected. It can also be thought that all estimates are asymptotically unbiased and consistent.

Now, we consider two cases to observe the performances of the estimates in the case of an outlier. In Case 1 (Case 2), we simulate the data, and we add 50 (70) on the maximum order statistics. The bias and MSEs of estimates in both cases are presented in Tables $3-4$. It is observed that the MSEs of $\widehat{\boldsymbol{\theta}}$ and $\widehat{\boldsymbol{\theta}}_{5}$ increase when the sample has an outlier. It is noticed that $\widehat{\beta}$ is less negatively affected by outliers than $\hat{\lambda}$ in terms of MSEs. In the presence of an outlier, the proportion estimate $\widehat{\boldsymbol{\theta}}_{3}$ performs better than any other estimate. The moment estimate is the estimator most negatively affected by outliers. Tables 1-2 show that $\widehat{\boldsymbol{\theta}}$ and $\widehat{\boldsymbol{\theta}}_{3}$ have almost the same performances based on the sample without an outlier. From Tables $3-4$, it is observed that $\widehat{\boldsymbol{\theta}}_{3}$ is robust and it has better performance than $\widehat{\boldsymbol{\theta}}$ based on a sample with an outlier.

Table 1. Average bias for selected parameters $\boldsymbol{\theta}$

\begin{tabular}{|c|c|c|c|c|c|c|c|c|c|c|c|c|}
\hline \multirow[b]{2}{*}{$n$} & \multicolumn{2}{|c|}{ True par. } & \multicolumn{10}{|c|}{ Estimators } \\
\hline & $\theta$ & & $\widehat{\theta}$ & & $\widehat{\boldsymbol{\theta}}_{2}$ & & $\widehat{\boldsymbol{\theta}}_{3}$ & & $\widehat{\boldsymbol{\theta}}_{4}$ & & $\widehat{\boldsymbol{\theta}}_{5}$ & \\
\hline 100 & 1.1 & 0.8 & -0.0108 & 0.0222 & -0.0129 & 0.0216 & -0.0124 & 0.0221 & -0.0129 & 0.0254 & -0.0111 & 0.0211 \\
\hline 200 & & & -0.0072 & 0.0177 & -0.0078 & 0.0166 & -0.0079 & 0.0168 & -0.0080 & 0.0192 & -0.0065 & 0.0175 \\
\hline 400 & & & -0.0062 & 0.0090 & -0.0066 & 0.0087 & -0.0068 & 0.0090 & -0.0066 & 0.0093 & -0.0063 & 0.0091 \\
\hline 600 & & & -0.0046 & 0.0112 & -0.0050 & 0.0113 & -0.0051 & 0.0113 & -0.0051 & 0.0122 & -0.0031 & 0.0105 \\
\hline 800 & & & -0.0041 & 0.0071 & -0.0043 & 0.0072 & -0.0042 & 0.0073 & -0.0044 & 0.0076 & -0.0039 & 0.0067 \\
\hline 1000 & & & -0.0035 & 0.0061 & -0.0036 & 0.0060 & -0.0035 & 0.0061 & -0.0036 & 0.0064 & -0.0035 & 0.0061 \\
\hline 100 & 0.9 & 0.7 & -0.0302 & 0.0224 & -0.0196 & 0.0234 & -0.02 & 0.0 & -0. & 0 . & -0.0 & 0.0070 \\
\hline 200 & & & -0.0181 & 0.0115 & -0.0144 & 0.0110 & -0.0176 & 0.0084 & -0.0123 & 0.0160 & -0.0181 & 0.0049 \\
\hline 400 & & & -0.0095 & 0.0078 & -0.0087 & 0.0060 & -0.0088 & 0.0056 & -0.0081 & 0.0084 & -0.0 & 0.0065 \\
\hline 600 & & & & & & & & 0.0088 & -0 . & & & 0.0085 \\
\hline 800 & & & & & & & & 69 & 63 & & & 0.0061 \\
\hline 1000 & & & -0.0045 & 0.0064 & -0.0057 & 0.0068 & -0.0055 & 0.0067 & -0.0054 & 0.0069 & -0.0046 & 0.0054 \\
\hline 100 & 1.5 & 5 & & & & & & & & & & \\
\hline 200 & & & & & & & & & & & & -0.0013 \\
\hline 400 & & & & & & & & & & 0.0052 & -0.0621 & 0.0003 \\
\hline 600 & & & -0.0001 & 0.0020 & 0.0000 & 0.0039 & -0.0001 & 0.0018 & 0.0003 & 0.0036 & -0. & 0.0007 \\
\hline 800 & & & 0002 & -0.0010 & 0.0002 & -0.0009 & 0.0002 & -0.0019 & 0.0006 & 0.0023 & -0.0625 & -0.0023 \\
\hline 1000 & & & 0.0001 & -0.0025 & 0.0003 & -0.0027 & 0.0002 & -0.0009 & 0.0006 & 0.0008 & -0.0543 & -0.0031 \\
\hline 100 & 0.4 & 5 & & & & & & & & & & \\
\hline 200 & & & -0.0001 & 0.0095 & 0.0004 & 0.0168 & 0.0005 & 0.0153 & -0.0001 & 0.0280 & 0.0036 & 0.0156 \\
\hline 400 & & & -0.0004 & -0.0052 & -0.0001 & -0.0021 & -0.0002 & 0.0021 & -0.0004 & 0.0086 & 0.0027 & 0.0023 \\
\hline 600 & & & -0.0002 & 0.0041 & 0.0002 & 0.0057 & 0.0002 & 0.0061 & 0.0001 & 0.0037 & 0.0020 & 0.0078 \\
\hline 800 & & & -0.0001 & 0.0041 & 0.0001 & 0.0054 & 0.0001 & 0.0054 & 0.0001 & 0.0017 & 0.0011 & 0.0072 \\
\hline 1000 & & & -0.0002 & 0.0012 & -0.0001 & 0.0029 & -0.0001 & 0.0017 & -0.0002 & 0.0014 & 0.0011 & 0.0041 \\
\hline
\end{tabular}


Table 2. Average MSEs for selected parameters $\theta$

\begin{tabular}{|c|c|c|c|c|c|c|c|c|c|c|c|c|}
\hline & \multicolumn{2}{|c|}{ True par. } & \multicolumn{10}{|c|}{ Estimators } \\
\hline$n$ & $\theta$ & & $\widehat{\boldsymbol{\theta}}$ & & $\widehat{\boldsymbol{\theta}}_{2}$ & & $\widehat{\boldsymbol{\theta}}_{3}$ & & $\widehat{\boldsymbol{\theta}}_{4}$ & & $\widehat{\boldsymbol{\theta}}_{5}$ & \\
\hline 100 & 1.1 & 0.8 & 0.0020 & 0.0075 & 0.0024 & 0.0092 & 0.0031 & 0.0101 & 0.0023 & 0.0084 & 0.0023 & 0.0071 \\
\hline 200 & & & 0.0010 & 0.0041 & 0.0013 & 0.0049 & 0.0013 & 0.0052 & 0.0012 & 0.0045 & 0.0008 & 0.0039 \\
\hline 400 & & & 0.0005 & 0.0020 & 0.0006 & 0.0023 & 0.0007 & 0.0025 & 0.0006 & 0.0021 & 0.0005 & 0.0019 \\
\hline 600 & & & 0.0003 & 0.0012 & 0.0004 & 0.0015 & 0.0004 & 0.0016 & 0.0004 & 0.0014 & 0.0002 & 0.0011 \\
\hline 800 & & & 0.0002 & 0.0009 & 0.0003 & 0.0011 & 0.0003 & 0.0012 & 0.0003 & 0.0010 & 0.0002 & 0.0008 \\
\hline 1000 & & & 0.0002 & 0.0007 & 0.0002 & 0.0009 & 0.0002 & 0.0009 & 0.0002 & 0.0008 & 0.0002 & 0.0007 \\
\hline 100 & 0.9 & 0.7 & 0.0056 & 0.0099 & 0.0071 & 0.0106 & 0.0089 & 0.0116 & 0.0060 & 0.0099 & 0.0203 & 0.0140 \\
\hline 200 & & & 0.0024 & 0.0052 & 0.0029 & 0.0056 & 0.0050 & 0.0067 & 0.0026 & 0.0054 & 0.0082 & 0.0064 \\
\hline 400 & & & 0.0006 & 0.0023 & 0.0011 & 0.0025 & 0.0016 & 0.0027 & 0.0009 & 0.0024 & 0.0014 & 0.0021 \\
\hline 600 & & & 0.0004 & 0.0015 & 0.0008 & 0.0016 & 0.0011 & 0.0017 & 0.0006 & 0.0016 & 0.0006 & 0.0013 \\
\hline 800 & & & 0.0003 & 0.0013 & 0.0006 & 0.0014 & 0.0006 & 0.0014 & 0.0005 & 0.0013 & 0.0005 & 0.0011 \\
\hline 1000 & & & 0.0002 & 0.0009 & 0.0004 & 0.0010 & 0.0005 & 0.0010 & 0.0004 & 0.0010 & 0.0004 & 0.0008 \\
\hline 100 & 1.5 & 5 & 0.0001 & 0.0498 & 0.0001 & 0.0 & 0.0 & 0.0617 & 0.0004 & 0. & 85 & 0.0531 \\
\hline 200 & & & 0.0000 & 0.0254 & 0.0001 & 0.0397 & 0.0001 & 0.0285 & 0.0002 & 0.0259 & 0.0524 & 0.0269 \\
\hline 400 & & & 0.0000 & 0.0128 & 0.0000 & 0.0208 & 0.0000 & 0.0129 & 0.0001 & 0.0124 & 0.0406 & 0.0133 \\
\hline 600 & & & 0.0000 & 0.0094 & 0.0000 & 0.0140 & 0.0000 & 0.0098 & 0.0000 & 0.0076 & 0.0424 & 0.0102 \\
\hline 800 & & & 0.0000 & 0.0060 & 0.0000 & 0.0105 & 0.0000 & 0.0071 & 0.0000 & 0.0055 & 0.0418 & 0.0063 \\
\hline 1000 & & & 0.0000 & 0.0050 & 0.0000 & 0.0078 & 0.0000 & 0.0052 & 0.0000 & 0.0038 & 0.0340 & 0.0053 \\
\hline 100 & 0.4 & 5 & 0.0001 & 0.0398 & 0.0002 & 0.0561 & 0.0002 & 0.0524 & 0.0009 & 0.0734 & 0.0005 & 0.0274 \\
\hline 200 & & & & & 0.0001 & & 0.0001 & 0.0204 & 0.0001 & 0.0219 & 0.0002 & 0.0146 \\
\hline 400 & & & 0.0000 & 0.0095 & 0.0000 & 0.0122 & 0.0000 & 0.0100 & 0.0001 & 0.0078 & 0.0001 & 0.0068 \\
\hline 600 & & & 0.0000 & 0.0066 & 0.0000 & 0.0084 & 0.0000 & 0.0065 & 0.0001 & 0.0038 & 0.0001 & 0.0049 \\
\hline 800 & & & 0.0000 & 0.0052 & 0.0000 & 0.0066 & 0.0000 & 0.0049 & 0.0000 & 0.0035 & 0.0000 & 0.0040 \\
\hline 1000 & & & 0.0000 & 0.0040 & 0.0000 & 0.0053 & 0.0000 & 0.0035 & 0.0000 & 0.0025 & 0.0000 & 0.0032 \\
\hline
\end{tabular}

Table 3. Average bias for some choice of parameter $\boldsymbol{\theta}=(0.9,10)$ when included an outlier sample

\begin{tabular}{|c|c|c|c|c|c|c|c|c|c|c|c|}
\hline \multirow[b]{2}{*}{$n$} & \multirow[b]{2}{*}{ Case } & \multicolumn{10}{|c|}{ Estimators } \\
\hline & & $\widehat{\theta}$ & & $\widehat{\boldsymbol{\theta}}_{2}$ & & $\widehat{\boldsymbol{\theta}}_{3}$ & & $\widehat{\boldsymbol{\theta}}_{4}$ & & $\widehat{\boldsymbol{\theta}}_{5}$ & \\
\hline 100 & 1 & -0.0029 & 0.4019 & 0.0001 & -0.0172 & -0.0002 & -0.0001 & 0.0010 & -0.0164 & -0.0037 & 1.7438 \\
\hline 200 & & -0.0030 & 0.1934 & -0.0002 & -0.0131 & -0.0002 & -0.0048 & 0.0003 & -0.0116 & 0.0209 & 0.9186 \\
\hline 400 & & -0.0018 & 0.0969 & 0.0001 & -0.0006 & 0.0000 & 0.0004 & 0.0006 & 0.0025 & 0.0247 & 0.4795 \\
\hline 600 & & -0.0011 & 0.0700 & 0.0000 & -0.0005 & 0.0000 & -0.0033 & 0.0004 & 0.0003 & 0.0099 & 0.3329 \\
\hline 800 & & -0.0008 & 0.0453 & -0.0002 & -0.0069 & -0.0001 & -0.0039 & 0.0001 & -0.0043 & 0.0085 & 0.2445 \\
\hline 1000 & & -0.0005 & 0.0403 & -0.0002 & 0.0013 & -0.0001 & 0.0008 & -0.0001 & 0.0014 & 0.0094 & 0.2015 \\
\hline 100 & 2 & -0.0005 & 0.5937 & -0.0002 & -0.0115 & -0.0004 & 0.0065 & 0.0001 & -0.0135 & -0.0304 & 2.8931 \\
\hline 200 & & -0.0006 & 0.3012 & -0.0002 & -0.0015 & -0.0002 & -0.0046 & 0.0005 & -0.0064 & 0.0212 & 1.5631 \\
\hline 400 & & -0.0002 & 0.1489 & 0.0000 & 0.0016 & 0.0001 & 0.0006 & 0.0005 & -0.0096 & 0.0227 & 0.8248 \\
\hline 600 & & -0.0001 & 0.1005 & -0.0001 & 0.0036 & 0.0000 & 0.0000 & 0.0001 & 0.0007 & 0.0299 & 0.5643 \\
\hline 800 & & -0.0001 & 0.0679 & 0.0000 & -0.0106 & -0.0001 & -0.0037 & 0.0002 & -0.0094 & 0.0194 & 0.4212 \\
\hline 1000 & & -0.0001 & 0.0591 & -0.0001 & -0.0024 & -0.0001 & -0.0016 & 0.0001 & 0.0027 & 0.0093 & 0.3446 \\
\hline
\end{tabular}

\section{Interval estimation for unknown distribution parameters}

In this section, asymptotically normal (AN) and uncorrected likelihood ratio (ULR) CIs are discussed for the parameters $\beta$ and $\lambda$. In general, the CIs of the distribution parameters are usually calculated by using a pivotal quantity based on MLEs of parameters. This method is well-known by statisticians and it is used without any hesitation. It is 
Table 4. Average MSEs for some choice of parameter $\boldsymbol{\theta}=(0.9,10)$ when included an outlier sample

\begin{tabular}{|c|c|c|c|c|c|c|c|c|c|c|c|}
\hline \multirow[b]{2}{*}{$n$} & \multirow[b]{2}{*}{ Case } & \multicolumn{10}{|c|}{ Estimators } \\
\hline & & $\widehat{\theta}$ & & $\widehat{\boldsymbol{\theta}}_{2}$ & & $\widehat{\boldsymbol{\theta}}_{3}$ & & $\widehat{\boldsymbol{\theta}}_{4}$ & & $\widehat{\boldsymbol{\theta}}_{5}$ & \\
\hline 100 & 1 & 0.0000 & 0.2566 & 0.0001 & 0.1907 & 0.0001 & 0.0848 & 0.0001 & 0.1011 & 0.0078 & 3.1280 \\
\hline 200 & & 0.0000 & 0.0870 & 0.0000 & 0.0862 & 0.0000 & 0.0352 & 0.0001 & 0.0446 & 0.0042 & 0.8910 \\
\hline 400 & & 0.0000 & 0.0350 & 0.0000 & 0.0424 & 0.0000 & 0.0170 & 0.0000 & 0.0218 & 0.0043 & 0.2557 \\
\hline 600 & & 0.0000 & 0.0223 & 0.0000 & 0.0308 & 0.0000 & 0.0130 & 0.0000 & 0.0167 & 0.0016 & 0.1283 \\
\hline 800 & & 0.0000 & 0.0150 & 0.0000 & 0.0236 & 0.0000 & 0.0086 & 0.0000 & 0.0122 & 0.0010 & 0.0731 \\
\hline 1000 & & 0.0000 & 0.0109 & 0.0000 & 0.0181 & 0.0000 & 0.0055 & 0.0000 & 0.0103 & 0.0011 & 0.0502 \\
\hline 100 & 2 & & & & & & 4 & & & & 8. \\
\hline 200 & & 0.0000 & 0.1386 & 0.0000 & 0.0919 & 0.0000 & 0.0407 & 0.0001 & 0.0539 & 0.0065 & 2.4860 \\
\hline 400 & & 0.0000 & 0.0462 & 0.0000 & 0.0426 & 0.0000 & 0.0174 & 0.0000 & 0.0228 & 0.0046 & 0.7034 \\
\hline 600 & & 0.0000 & 0.0262 & 0.0000 & 0.0307 & 0.0000 & 0.0124 & 0.0000 & 0.0166 & 0.0055 & 0.3347 \\
\hline 800 & & 0.0000 & 0.0171 & 0.0000 & 0.0205 & 0.0000 & 0.0078 & 0.0000 & 0.0108 & 0.0034 & 0.1898 \\
\hline 1000 & & 0.0000 & 0.0141 & 0.0000 & 0.0177 & 0.0000 & 0.0060 & 0.0000 & 0.0104 & 0.0016 & 0.1291 \\
\hline
\end{tabular}

established that, under some conditions, the AN of MLEs can be formulated as

$$
\widehat{\boldsymbol{\theta}} \stackrel{d}{\rightarrow} N_{2}\left(\boldsymbol{\theta}, \mathbb{I}^{-1}(\boldsymbol{\theta})\right),
$$

where $\widehat{\boldsymbol{\theta}}$ is the MLE of $\boldsymbol{\theta}$ given in (5.2) and $\mathbb{I}(\boldsymbol{\theta})$ is Fisher Information matrix. This matrix can be estimated by negative Hessian matrix at $\widehat{\boldsymbol{\theta}}$ and it is denoted by $\widehat{\mathbb{I}}(\widehat{\boldsymbol{\theta}})$. Using this result, the $100 \times(1-\alpha) \%$ AN CIs of theparameters $\beta$ and $\lambda$ are constructed, respectively, by

$$
\begin{aligned}
& \widehat{\beta} \pm z_{1-\frac{\alpha}{2}} \times s e(\widehat{\beta}), \\
& \widehat{\lambda} \pm z_{1-\frac{\alpha}{2}} \times s e(\widehat{\lambda}),
\end{aligned}
$$

where $z_{a}$, is the $a^{\text {th }}$ quantile of the standard normal distribution, se $(\widehat{\beta})$ and $s e(\widehat{\lambda})$ are the roots of the diagonal member of $\widehat{\mathbb{I}}^{-1}(\widehat{\boldsymbol{\theta}})$ and the $s e(\cdot)$ stands for standard error of an estimate.

By the way, there is another method called ULR, which is not used in most of the statistical software, but it has interesting properties. This method can be described as follows: Under usual regularity assumptions on the likelihood function, if the $\beta$ is true parameter, then $-2 \log (\ell(\beta, \widetilde{\lambda})-\ell(\widehat{\boldsymbol{\theta}}))$ distributed as $\chi^{2}$ with degrees of freedom 1 , where $\lambda$ is the nuisance parameters, $\ell$ is the log-likelihood function as in (5.1), $\widehat{\boldsymbol{\theta}}$ is the MLE of $\boldsymbol{\theta}$ given in (5.2) and $\widetilde{\lambda}$ is the restricted MLEs of $\lambda$ given a fixed value of $\beta$. Using this fact, $100 \times(1-\alpha) \%$ ULR CI limits $\left(\beta_{L}, \beta_{U}\right)$ that satisfy

$$
\ell(\beta, \widetilde{\lambda})=\ell(\widehat{\boldsymbol{\theta}})-\frac{1}{2} \chi_{(1)}^{2}(1-\alpha)
$$

with $\beta_{L}<\hat{\beta}$ and $\beta_{U}>\hat{\beta}$, where $\chi_{(1)}^{2}(a)$ is the $a^{\text {th }}$ quantile of the $\chi^{2}$ distribution with 1 degree of freedom. The $100 \times(1-\alpha) \%$ ULR CIs can be produced in the same manner for $\lambda$.

Due to Fraser [8], the ULR and AN CIs are asymptotically equivalent. The ULR CIs are transformation invariant, unlike AN method. ULR CIs always produce limits inside of the parameter space. There is no need to calculate the variances of the estimates using second derivatives of likelihood, unlike to AN. 


\subsection{Simulation study for the CIs}

In this simulation study, 2000 trials are conducted to predict the overage probabilities(CPs) of the AN and ULR CIs. The BFGS method is used in all maximization procedures. The nominal level is fixed at 0.95. To obtain CPs of ULR CIs, there is no need to get the CIs limits. It is possible that the CPs of ULR CIs can be simulated by a likelihood ratio test on the true parameter. In this case, the simulation can be completed in a short time. The simulated CPs of these intervals are presented in Table 5 for $\boldsymbol{\theta}=(0.5,2),(1.2,1),(0.2,10)$ and $(1.5,4)$. From Table 5 , we observe that the CPs of the ULRs and AN CIs reach the desired level, even for small samples.

Table 5. The CPs of AN and ULR CIs

\begin{tabular}{|c|c|c|c|c|c|}
\hline & & $\mathrm{AN}$ & & ULR & \\
\hline$\theta$ & $n$ & $\beta$ & $\lambda$ & $\beta$ & $\lambda$ \\
\hline$(0.5,2)$ & 50 & 0.9545 & 0.9410 & 0.9495 & 0.9475 \\
\hline & 100 & 0.9610 & 0.9400 & 0.9540 & 0.9415 \\
\hline & 250 & 0.9545 & 0.9480 & 0.9495 & 0.9480 \\
\hline & 500 & 0.9595 & 0.9485 & 0.9545 & 0.9500 \\
\hline & 1000 & 0.9510 & 0.9495 & 0.9510 & 0.9485 \\
\hline$(1.2,1)$ & 50 & 0.9580 & 0.9665 & 0.9560 & 0.9520 \\
\hline & 100 & 0.9550 & 0.9665 & 0.9525 & 0.9530 \\
\hline & 250 & 0.9530 & 0.9585 & 0.9490 & 0.9520 \\
\hline & 500 & 0.9485 & 0.9540 & 0.9460 & 0.9495 \\
\hline & 1000 & 0.9440 & 0.9575 & 0.9435 & 0.9540 \\
\hline$(0.2,10)$ & 50 & 0.9595 & 0.9630 & 0.9540 & 0.9520 \\
\hline & 100 & 0.9530 & 0.9480 & 0.9515 & 0.9435 \\
\hline & 250 & 0.9530 & 0.9575 & 0.9490 & 0.9545 \\
\hline & 500 & 0.9505 & 0.9515 & 0.9480 & 0.9505 \\
\hline & 1000 & 0.9520 & 0.9490 & 0.9510 & 0.9480 \\
\hline$(1.5,4)$ & 50 & 0.9330 & 0.9435 & 0.9405 & 0.9475 \\
\hline & 100 & 0.9440 & 0.9445 & 0.9490 & 0.9415 \\
\hline & 250 & 0.9545 & 0.9495 & 0.9580 & 0.9530 \\
\hline & 500 & 0.9490 & 0.9480 & 0.9485 & 0.9485 \\
\hline & 1000 & 0.9405 & 0.9570 & 0.9405 & 0.9580 \\
\hline
\end{tabular}

\section{Modeling practical data with analysis}

In this section, we present data modeling analysis for the number of strike outbreaks in the coal mining industry in the UK. The data set of this number is given by Table 6 . It was analyzed by Castillo and Casany [6], noting that they reported it from Kendall [9]. For the comparison issue, we consider some well-known discrete distributions, such as geometric, Poisson and negative binomial distributions. The MLEs of the parameters, the chi-square test statistics with $p$-values, AIC, Bayesian information criterion (BIC), corrected Akaike's information criterion (CAIC) and HannanQuinn information criterion (HQIC) are given in Table 7. From Table 7, the best model is the CosPois distribution according to all the criteria.

The $95 \%$ ULR CIs of $\beta$ and $\lambda$ are given, respectively, by $(0.1504693,0.3675793)$ and $(1.021822,1.704162)$. It is noticed that $\mathrm{R}$ functions $\mathbf{n l m}$ and uniroot are used to get these intervals. 
Table 6. Number of strike outbreaks in the coal mining industry in the UK

\begin{tabular}{ccccccc}
\hline Count & 0 & 1 & 2 & 3 & 4 & Total \\
\hline Observed frequencies & 46 & 76 & 24 & 9 & 1 & 156 \\
\hline
\end{tabular}

Table 7. Distribution modeling results for the UK coal data

\begin{tabular}{crrrr}
\hline & CosPois & Geometric & Poisson & Negative Binomial \\
\hline$\ell$ & -189.1211 & -215.5672 & -191.9362 & -191.9723 \\
$-2 \ell$ & 378.2422 & 431.1343 & 383.8724 & 383.9446 \\
$\mathrm{AIC}$ & 382.2422 & 433.1343 & 385.8724 & 387.9446 \\
$\mathrm{BIC}$ & 388.3419 & 436.1842 & 388.9223 & 394.0443 \\
$\mathrm{CAIC}$ & 382.3206 & 433.1603 & 385.8984 & 388.0230 \\
$\mathrm{HQIC}$ & 384.7196 & 434.3731 & 387.1111 & 390.4220 \\
$\widehat{\beta}$ & 0.3081 & 0.5016 & 0.9935 & 0.0017 \\
$\widehat{\lambda}$ & 1.3630 & & & 572.1488 \\
\hline Chi-Square & 5.0785 & 134.6523 & 9.9362 & 9.4912 \\
\hline $\mathrm{df}$ & 2 & 3 & 3 & 2 \\
\hline$p$ value & 0.0789 & 0.0000 & 0.0191 & 0.0020 \\
\hline
\end{tabular}

The observed Fisher information matrix is computed by

$$
\widehat{\mathbb{I}}^{-1}(\widehat{\boldsymbol{\theta}})=\left(\begin{array}{cc}
0.001867 & 0.005376 \\
0.005376 & 0.03037
\end{array}\right) .
$$

Using (6.1)-(6.2) and (7.1), the 95\% AN CIs of $\beta$ and $\lambda$ are given, respectively, by $(0.2234658,0.3928478)$ and $(1.021510,1.704632)$.

In order to observe the robustness property of the proportion type estimates, we include a value "100" with frequency 1 as an outlier in the practical data and we refer to this data as "modified data". All the estimates given in (5.2)-(5.5) and (5.8) based on practical data and modified data (practical data with an outlier) are given in Table 8 . The fitted and observed frequencies are also presented in Figures 3-4 based on practical data and modified data. It is noticed that the fitted frequencies are calculated based the on estimates $\widehat{\boldsymbol{\theta}}$ and $\widehat{\boldsymbol{\theta}}_{3}$. From Figure 3, it is observed that there is no significant difference between the observed and fitted frequencies when the data do not contain an outlier. From Figure 4, it can be concluded that the fitted frequencies with estimate $\widehat{\boldsymbol{\theta}}_{3}$ based on the modified data do not change and they are compatible with the observed frequencies. However, it is also observed from Figure 4 that the fitted frequencies with estimate $\widehat{\boldsymbol{\theta}}$ based on the modified data are negatively affected by an outlier. Table 8 shows that $\widehat{\boldsymbol{\theta}}_{3}$ is not affected by an outlier unlike $\widehat{\boldsymbol{\theta}}$. In addition, while the estimate $\widehat{\boldsymbol{\theta}}_{3}$ is almost unaffected by the presence of an outlier, the estimate $\widehat{\boldsymbol{\theta}}$ is negatively affected by the presence of an outlier. In this discussion, we conclude that the proportion type estimate $\widehat{\boldsymbol{\theta}}_{3}$ can be a good robust alternative to $\widehat{\boldsymbol{\theta}}$.

Table 8. All estimates for $\boldsymbol{\theta}$ under practical and modified data

\begin{tabular}{lccccc}
\hline & $\widehat{\boldsymbol{\theta}}$ & $\widehat{\boldsymbol{\theta}}_{2}$ & $\widehat{\boldsymbol{\theta}}_{3}$ & $\widehat{\boldsymbol{\theta}}_{4}$ & $\widehat{\boldsymbol{\theta}}_{5}$ \\
\hline Based on UK coal data & $(0.3081,1.3630)$ & $(0.4669,1.8075)$ & $(0.4485,1.8562)$ & $(0.4400,1.7266)$ & $(0.2987,1.3037)$ \\
& & & & & \\
Based on modified data & $(0.3730,2.5052)$ & $(0.4687,1.8187)$ & $(0.4492,1.8676)$ & $(0.4257,1.6041)$ & $(0.0272,7.3394)$ \\
\hline
\end{tabular}




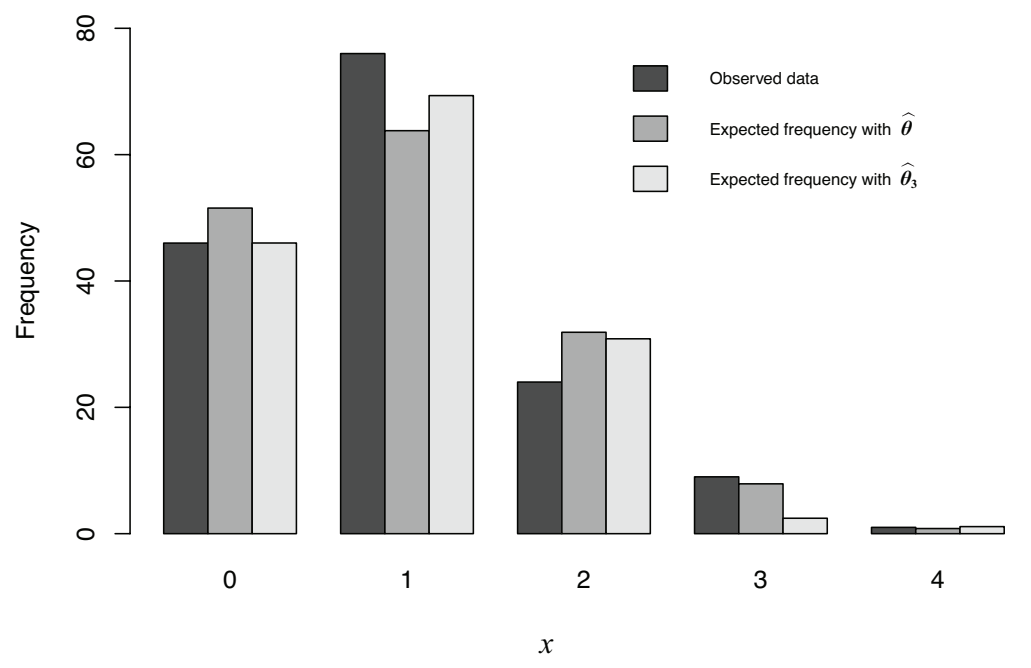

Figure 3. Observed and fitted frequencies with $\widehat{\boldsymbol{\theta}}$ and $\widehat{\boldsymbol{\theta}}_{3}$ for UK coal data

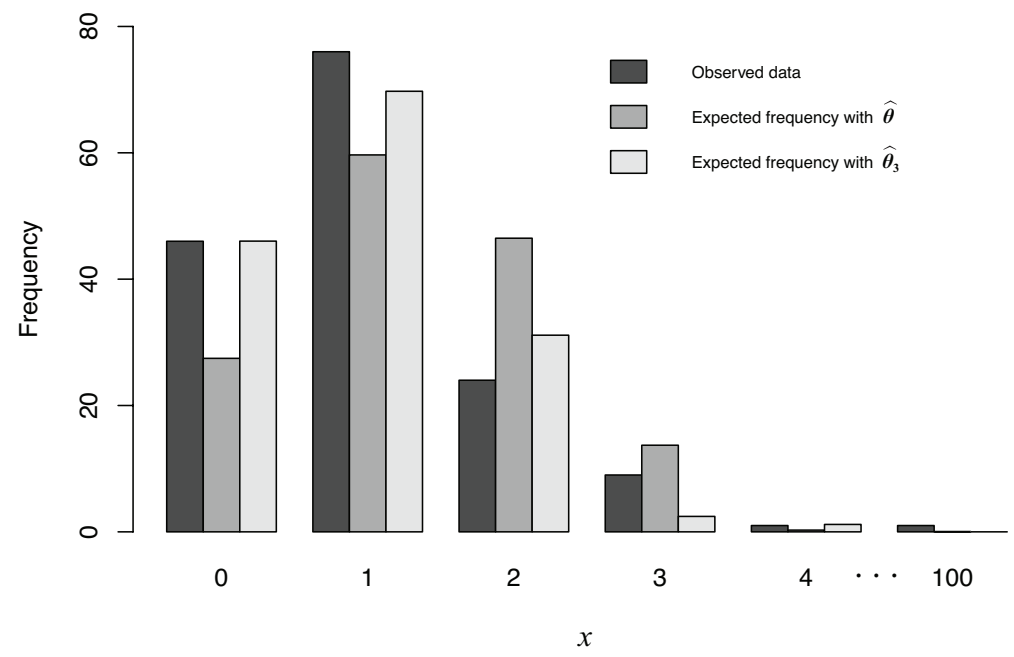

Figure 4. Observed and fitted frequencies with $\widehat{\boldsymbol{\theta}}$ and $\widehat{\boldsymbol{\theta}}_{3}$ based on modified data(included an outlier to practical data)

\section{Count regression analysis}

In many disciplines, the researchers investigate the effects of covariates on the response variable. If the response is observed by counting, ordinary regression analysis can not be applied to the data. In this case, count regression analysis should be used to get a decision on the covariates effects on the response. The Poisson and negative binomial regression models are most popular in count regression analysis. The Poisson regression model can not be used when the count data have an inflated number of zeros. The zeroinflated Poisson, the zero-inflated negative binomial, Hurdle-Poisson and Hurdle-negative binomial regression models can be used for analyzing this type of data. In this section, we provide a count regression analysis by using the CosPois distribution to be an alternative the popular ones. 
Let us define a random variable

$$
Y=\left\{\begin{array}{cc}
0, & U=0 \\
X, & U=1
\end{array}\right.
$$

where $U$ is Bernoulli random variable with success probability $1-\pi$ and $X$ is the $\operatorname{CosPois}(\boldsymbol{\theta})$ random variable as well as $U$ and $X$ are independent. Then, the zero-inflated CosPois distribution defined by pmf of random variable $Y$ in (8.1)

$$
P(Y=y)=\left\{\begin{array}{cc}
\pi+(1-\pi) C_{\beta, \lambda}, & y=0 \\
(1-\pi) C_{\beta, \lambda}[\cos (\beta y)]^{2} \frac{\lambda^{y}}{y !}, & y>0,
\end{array}\right.
$$

where $\pi \in(0,1)$ and it is denoted by $\mathrm{ZICosPois}(\beta, \lambda, \pi)$. It is noticed that the random variable given in (8.1) can be represented by $Y=U X$, where $U$ and $X$ are independent random variables from $\operatorname{Bernoulli}(1-\pi)$ and $\operatorname{CosPois}(\boldsymbol{\theta})$, respectively. Using this relation, the mean and variance $\mathrm{ZICosPois}(\beta, \lambda, \pi)$ distribution are obtained by

$$
E(Y)=(1-\pi) \mu
$$

and

$$
\operatorname{Var}(Y)=(1-\pi)\left(\sigma^{2}+\mu^{2} \pi\right)
$$

respectively, where $\mu$ and $\sigma^{2}$ are mean and variance of $\operatorname{CosPois}(\boldsymbol{\theta})$ given in (3.2) and (3.3).

Let $Y_{i},(i=1,2, \ldots, n)$ be a ZICosPois $\left(\lambda_{i}, \beta, \pi_{i}\right)$ random variable with pmf given in (8.2) and $Y_{1}, Y_{2}, \ldots, Y_{n}$ be independent. The ZICosPois count regression model is given by the following functional expressions:

$$
\log \left(\lambda_{i}\right)=\boldsymbol{x}_{i}^{\mathrm{T}} \boldsymbol{\beta}
$$

and

$$
\log \left(\frac{\pi_{i}}{1-\pi_{i}}\right)=\boldsymbol{z}_{i}^{\mathrm{T}} \boldsymbol{\gamma}
$$

where $\boldsymbol{\beta}=\left(\beta_{1}, \beta_{2}, \ldots, \beta_{p}\right)^{\mathrm{T}} \in \mathbb{R}^{p}, \boldsymbol{\gamma}=\left(\gamma_{1}, \gamma_{2}, \ldots, \gamma_{q}\right)^{\mathrm{T}} \in \mathbb{R}^{q}, p+q+1<n$. In addition, $\boldsymbol{x}_{i}^{\mathrm{T}}=\left(x_{i 1}, x_{i 2}, \ldots, x_{i p}\right)$ and $\boldsymbol{z}_{i}^{\mathrm{T}}=\left(z_{i 1}, z_{i 2}, \ldots, z_{i q}\right)$ are known values on covariates. Moreover, we assume that the matrices $\boldsymbol{X}=\left(\boldsymbol{x}_{1}, \boldsymbol{x}_{2}, \ldots, \boldsymbol{x}_{p}\right)^{\mathrm{T}}$ and $\boldsymbol{Z}=\left(\boldsymbol{z}_{1}, \boldsymbol{z}_{2}, \ldots, \boldsymbol{z}_{q}\right)^{\mathrm{T}}$ have rank $p$ and $q$, respectively. It should be take $x_{i 1}=z_{i 1}=1$ (for $i=1, \ldots, n$ ) to include intercepts in a model. It is noticed that the covariates in $\boldsymbol{Z}$ can be a subset of the covariates in $\boldsymbol{X}$.

\subsection{Parameter estimation on the ZICosPois regression parameters}

Let $Y_{1}, Y_{2}, \ldots, Y_{n}$ be $n$ independent random variables but non identically distributed as ZICosPois $\left(\beta, \lambda_{1}, \pi_{1}\right)$, ZICosPois $\left(\beta, \lambda_{2}, \pi_{2}\right), \ldots$, ZICosPois $\left(\beta, \lambda_{n}, \pi_{n}\right)$, respectively. Using the expressions given in (8.3)-(8.4) and the pmf given by (8.2), the log-likelihood function can be written by

$$
\begin{aligned}
\ell(\phi) \propto \sum_{i \in\left\{j: y_{j}=0, j=1,2, \ldots, n\right\}} \log \left(\pi_{i}+\left(1-\pi_{i}\right) C_{\beta, \lambda_{i}}\right)+ \\
\quad \sum_{i \in\left\{j: y_{j}>0, j=1,2, \ldots, n\right\}}\left\{\log \left(1-\pi_{i}\right)+\log \left(C_{\beta, \lambda_{i}}\right)+2 \log \left[\cos \left(\beta y_{i}\right)\right]+y_{i} \log \left(\lambda_{i}\right)\right\},
\end{aligned}
$$

where $\phi=(\beta, \gamma, \beta)$,

$$
\pi_{i}=\frac{\exp \left(\boldsymbol{z}_{i}^{\mathrm{T}} \boldsymbol{\gamma}\right)}{1+\exp \left(\boldsymbol{z}_{i}^{\mathrm{T}} \boldsymbol{\gamma}\right)}
$$


and

$$
\lambda_{i}=\exp \left(\boldsymbol{x}_{i}^{\mathrm{T}} \boldsymbol{\beta}\right)
$$

for $i=1, \ldots, n$. The MLE of $\phi$ (it is denoted by $\widehat{\phi}$ ) can be defined by

$$
\widehat{\phi}=\underset{\phi}{\arg \max }(\ell(\phi))
$$

and it can be obtained by optim function in $\mathrm{R}$ with different algorithms. Under some mild regularity conditions

$$
\widehat{\phi} \stackrel{d}{\rightarrow} N_{p+q+1}\left(\phi, \mathbb{I}^{-1}(\phi)\right),
$$

where $\ell(\phi)$ is $\log$-likelihood given in $(8.5)$ and $\mathbb{I}(\cdot)$ is Fisher information matrix. This matrix it can be estimated by the negative Hessian matrix of $\ell(\phi)$ at $\widehat{\phi}$. Using the existing theory, the approximate CIs for all regression parameters can easily be constructed.

\subsection{Goodness-of-fit and residuals analysis}

Evaluating a fitted model is an important part of data analysis, especially in regression models, and residual analysis is a useful tool to validate a fitted model. The presence of outlier observations, model misspecifications or incorrect specification of the error distribution can be detected by residuals. In this regard, it is important to diagnose the abnormal residuals in count regression models with over-dispersed or under-dispersed model. However, the distribution of error terms are unknown in count regression analysis and the classical normal QQ-plots do not work in these models. Fortunately, [2] suggests a methodology to construct the simulated envelopes for unknown distributed residuals. In order to perform the residual analysis, the following standardized ordinary residuals (Pearson residuals) can be used:

$$
e_{i}=\frac{\left(y_{i}-\widehat{E}\left(Y_{i} \mid \boldsymbol{X}, \boldsymbol{Z}\right)\right)}{\widehat{\operatorname{Var}}\left(Y_{i} \mid \boldsymbol{X}, \boldsymbol{Z}\right)}, i=1,2, \ldots, n
$$

with

$$
\begin{gathered}
\widehat{E}\left(Y_{i} \mid \boldsymbol{X}, \boldsymbol{Z}\right)=\left(1-\widehat{\pi}_{i}\right) \widehat{\mu}_{i}, \\
\widehat{\operatorname{Var}}\left(Y_{i} \mid \boldsymbol{X}, \boldsymbol{Z}\right)=\left(1-\widehat{\pi}_{i}\right)\left(\sigma^{2}+\widehat{\mu}_{i}^{2} \widehat{\pi}_{i}\right), \\
\widehat{\mu}_{i}=\widehat{\lambda}_{i} \frac{1+e^{-\widehat{\lambda}_{i}[1-\cos (2 \widehat{\beta})]} \cos \left[\widehat{\lambda}_{i} \sin (2 \widehat{\beta})+2 \widehat{\beta}\right]}{1+e^{-\widehat{\lambda}_{i}[1-\cos (2 \widehat{\beta})]} \cos \left[\widehat{\lambda}_{i} \sin (2 \widehat{\beta})\right]}, \\
\widehat{\pi}_{i}=\frac{\exp \left(\boldsymbol{z}_{i}^{\mathrm{T}} \widehat{\gamma}\right)}{1+\exp \left(\boldsymbol{z}_{i}^{\mathrm{T}} \widehat{\gamma}\right)},
\end{gathered}
$$

and

$$
\widehat{\lambda}_{i}=\exp \left(\boldsymbol{x}_{i}^{\mathrm{T}} \widehat{\boldsymbol{\beta}}\right),
$$

where $\widehat{\boldsymbol{\beta}}, \widehat{\gamma}$ and $\widehat{\beta}$ denote the MLE of $\boldsymbol{\beta}, \boldsymbol{\gamma}$ and $\beta$, respectively. Since the distribution of the standardized residuals are unknown, the methodology proposed by Atkinson [2] methodology is used to construct the simulated envelope. Simulated envelopes can be used to detect the incorrect specification of the error distribution or the presence of possible outliers. The observations corresponding to the residuals outside of the simulated envelope should be re-examined. Furthermore, if a considerable proportion of points falls outside the envelope, then there is evidence against the adequacy of the fitted model. 


\subsection{Count regression example with the ZICosPois distribution}

In this subsection, an example with practical data is provided to show the applicability of the CosPois distribution to count regression. We illustrate the application of CosPois regression by considering the data set obtained from the Journal of Applied Econometrics 1997. The data was obtained from the survey conducted by the Australian Health Survey between 1977-1978. The data set is available at: http://qed.econ.queensu.ca/jae/1997v12.3/cameron-johansson/.

In this example, the number of appointments in the past 2 weeks with a doctor or specialist is considered as a response variable (doctorco). We interest to model the response variable by using the following 12 covariates:

(a) Four socioeconomic variables: sex, age, agesq, income,

(b) Three health insurance status indicators: levyplus, freepoor, freerepa,

(c) Two recent health status measures: illness, actdays,

(d) Three long-term health status measures: hscore, chcond1, chcond2.

Details for these 12 covariates can be found in Cameron et al. [5]. The high proportion of zeros (about 80\%) leading to acceptance of the proposed ZICosPois model (see Figure 5 ). We give the results on this data by the previously proposed models, such as negative binomial, zero-inflated Poisson, zero-inflated negative binomial, zero-inflated geometric, Hurdle-Poisson and Hurdle-negative binomial regression model beside our model. Because they are suitable choices for regression modeling when the response variable contains zeros with high frequency. It is noticed that the $\mathrm{R}$ functions zeroinfl and hurdle (in library pscl) can be used to perform these all count regression analyses. Tables 9-10 present the maximum likelihood estimates of all models, including an intercept. The $\ell(\widehat{\theta})$, AIC and BIC are also presented in these tables to compare the models. Tables 9-10 show that ZICospois regression model is superior over the other well-known count regression models in terms of all criteria.

The QQ-plots and simulated envelopes for the Pearson residuals of the ZICospois, ZIP and ZINB regression models are given in Figure 6. The lines in the graphics represent the 5th percentile, the mean, and the 95th percentile of 500 simulated points for each observation. Simulated envelopes for the Pearson residuals under ZICosPois model are plotted by using the algorithm in Lemonte et al. [12]. This algorithm is also adopted from Atikson [2]. The other simulated envelops are plotted by the $\mathrm{R}$ function hnp in library hnp. In Figure 6, the observation outside of the envelopes are presented with red points. The proportion of red points are $0.40462 \%, 3.4104 \%$ and $5.7225 \%$ for ZICospois, ZIP and ZINB regression models, respectively. Figure 6 demonstrates that the ZICosPois regression model provides a better fit to the survey data set than the ZIP and the ZINB regression models. Moreover, there is no evidence of lacks of fit for the ZICosPois model. The high proportion of points falls outside the envelopes against the adequacy of the fitted ZIP and the ZINB regression models.

\section{Concluding remarks}

In this paper, a new discrete distribution is introduced. Some distributional properties are discussed. The moment generation function, the expected value and variance of the new distribution are derived in explicit form. Some estimators are suggested to estimate the model parameters. It is observed that one of the proportion type estimates for the CosPois parameters is a competitor with MLEs based on a sample without an outlier. It is also observed that the proportion type estimates have a robustness property when an outlier is being in a sample. A new count regression analysis is also introduced using a zeroinflation framework. The applicability of our count regression analysis is demonstrated based on practical data. 


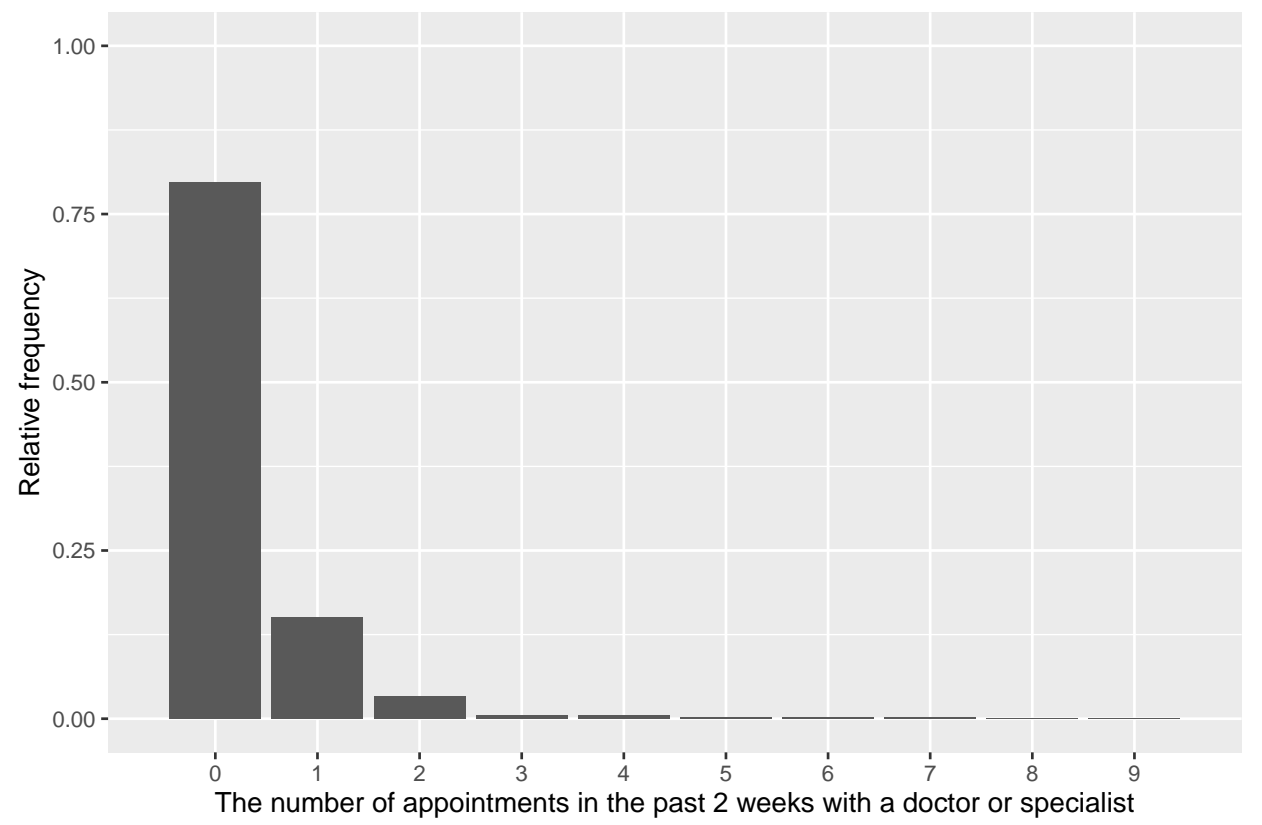

Figure 5. Relative frequency of response variable

Table 9. The MLEs with standard errors (SEs) of zero-inflated CosPois, Poisson and negative binomial models parameters based on 1977-1978 data set from the Australian Health Survey

\begin{tabular}{|c|c|c|c|c|c|c|c|c|c|}
\hline & \multicolumn{3}{|c|}{ Zero-inflated CosPois } & \multicolumn{3}{|c|}{ Zero-inflated Poisson } & \multicolumn{3}{|c|}{ Zero-inflated negative binomial model } \\
\hline & Estimate (SE) & $z$ value & $p$ value & Estimate (SE) & $z$ value & $p$ value & Estimate (SE) & $z$ value & $p$ value \\
\hline (Intercept) & $-0.1484(0.2210)$ & -0.671 & 0.5019 & $-1.0503(0.2550)$ & -4.118 & 0.0000 & $-1.2334(0.2956)$ & -4.172 & 0.0000 \\
\hline Sex & $-0.0185(0.0613)$ & -0.302 & 0.7628 & $-0.0269(0.0715)$ & -0.377 & 0.7059 & $0.0102(0.0838)$ & 0.123 & 0.9023 \\
\hline Age & $2.3269(1.0968)$ & 2.122 & 0.0339 & $3.1283(1.2970)$ & 2.412 & 0.0158 & $2.1026(1.5414)$ & 1.364 & 0.1725 \\
\hline Agesq & $-2.5469(1.1609)$ & -2.194 & 0.0282 & $-3.4092(1.3735)$ & -2.482 & 0.0130 & $-2.1868(1.6389)$ & -1.334 & 0.1821 \\
\hline Income & $-0.2183(0.0974)$ & -2.241 & 0.0250 & $-0.2949(0.1129)$ & -2.612 & 0.0090 & $-0.2142(0.1326)$ & -1.615 & 0.1064 \\
\hline Levyplus & $-0.0351(0.0813)$ & -0.432 & 0.6659 & $-0.0337(0.0964)$ & -0.350 & 0.7263 & $-0.0950(0.1144)$ & -0.831 & 0.4060 \\
\hline Freepoor & $-0.2337(0.2105)$ & -1.110 & 0.2669 & $-0.3769(0.2389)$ & -1.578 & 0.1146 & $-0.4812(0.2825)$ & -1.703 & 0.0886 \\
\hline Freerepa & $-0.1775(0.0990)$ & -1.793 & 0.0730 & $-0.2152(0.1171)$ & -1.837 & 0.0662 & $-0.1894(0.1401)$ & -1.351 & 0.1766 \\
\hline Illness & $0.0295(0.0205)$ & 1.439 & 0.1501 & $0.0486(0.0245)$ & 1.978 & 0.0478 & $0.0515(0.0293)$ & 1.753 & 0.0795 \\
\hline Actdays & $0.0516(0.0052)$ & 9.923 & 0.0000 & $0.0826(0.0059)$ & 13.943 & 0.0000 & $0.1038(0.0077)$ & 13.314 & 0.0000 \\
\hline Hscore & $0.0101(0.0096)$ & 1.052 & 0.2928 & $0.0178(0.0113)$ & 1.579 & 0.1143 & 00234(0.0137) & 1.711 & 0.0871 \\
\hline Chcond1 & $-0.0153(0.0801)$ & -0.191 & 0.8485 & $-0.0133(0.0923)$ & -.0145 & 0.8848 & $-0.0002(0.1080)$ & -0.0003 & 0.9979 \\
\hline Chcond2 & $-0.0375(0.0885)$ & -0.424 & 0.6718 & $-0.0340(0.1027)$ & -0.332 & 0.7401 & $0.0549(0.1206)$ & 0.455 & 0.6490 \\
\hline$\beta$ & $2.6821(0.0050)$ & 536.420 & 0.0000 & & & & $0.5484(0.1377)$ & 3.981 & 0.0000 \\
\hline & & & Zero-infl & efficie & (binol & ith & t link): & & \\
\hline (Intercept) & $1.4271(0.4412)$ & 3.235 & 0.0012 & $0.7863(0.5717)$ & 1.375 & 0.1692 & $0.6218(0.7525)$ & 0.826 & 0.4086 \\
\hline Sex & $-0.3751(0.1307)$ & -2.870 & 0.0041 & $-0.4884(0.1714)$ & -2.848 & 0.0043 & $-0.5918(0.2283)$ & -2.592 & 0.0095 \\
\hline Age & $7.1672(2.4945)$ & 2.873 & 0.0041 & 10.4961(3.2709) & 3.209 & 0.0013 & $10.6764(4.3860)$ & 2.434 & 0.0149 \\
\hline Agesq & $-9.3217(2.7833)$ & -3.349 & 0.0008 & $-13.3374(3.6899)$ & -3.615 & 0.0003 & $-13.8207(5.0017)$ & -2.763 & 0.0057 \\
\hline Income & $-0.2169(0.2045)$ & -1.061 & 0.2889 & $-0.4366(0.2645)$ & -1.651 & 0.0987 & $-0.3653(0.3459)$ & -1.056 & 0.2910 \\
\hline Levyplus & $-0.4012(0.1539)$ & -2.607 & 0.0091 & $-0.4331(0.1967)$ & -2.202 & 0.0276 & $-0.6401(0.2642)$ & -2.422 & 0.0154 \\
\hline Freepoor & $0.5536(0.4121)$ & 1.343 & 0.1792 & $0.3080(0.5078)$ & 0.607 & 0.5441 & $0.1106(0.6590)$ & 0.168 & 0.8666 \\
\hline Freerepa & $-0.8847(0.2227)$ & -3.973 & 0.0001 & $-1.1490(0.3049)$ & -3.768 & 0.0001 & $-1.3751(0.4473)$ & -3.074 & 0.0021 \\
\hline Illness & $-0.3477(0.0553)$ & -6.288 & 0.0000 & $-0.4158(0.0807)$ & -5.150 & 0.0000 & $-0.6716(0.1560)$ & -4.303 & 0.0000 \\
\hline Actdays & $-1.0338(0.1494)$ & -6.920 & 0.0000 & $-1.2560(0.2380)$ & -5.275 & 0.0000 & $-1.7873(0.6531)$ & -2.736 & 0.0062 \\
\hline Hscore & $-0.1016(0.0300)$ & -3.387 & 0.0007 & $-0.0974(0.0385)$ & -2.528 & 0.0114 & $-0.1045(0.0558)$ & -1.872 & 0.0616 \\
\hline Chcond1 & $-0.1313(0.1469)$ & -0.894 & 0.3714 & $-0.1271(0.1990)$ & -0.639 & 0.5229 & $-0.1190(0.2792)$ & -0.426 & 0.6698 \\
\hline Chcond2 & $-0.5241(0.2239)$ & -2.341 & 0.0192 & $-0.6037(0.3060)$ & 1.973 & 0.0485 & $-0.4889(0.4144)$ & -1.180 & 0.2380 \\
\hline$\ell$ & -3104.838 & & & -3174.185 & & & -3107.593 & & \\
\hline $\mathrm{AIC}$ & 6263.677 & & & 6400.370 & & & 6269.187 & & \\
\hline $\mathrm{BIC}$ & 6440.648 & & & 6570.787 & & & 6446.158 & & \\
\hline
\end{tabular}


Table 10. The MLEs with standard errors (SEs) of zero-Hurdle and zero-inflated geometric models parameters based on 1977-1978 Australian Health Survey data set

\begin{tabular}{|c|c|c|c|c|c|c|c|c|c|}
\hline & \multicolumn{3}{|c|}{ Zero-inflated geometric } & \multicolumn{3}{|c|}{ Zero-Hurdle-Poisson model } & \multicolumn{3}{|c|}{ Zero-Hurdle-negative binomial model } \\
\hline & Estimate (SE) & $z$ value & $p$ value & Estimate (SE) & $z$ value & $p$ value & Estimate (SE) & $z$ value & $p$ value \\
\hline (Intercept) & $-1.3991(0.2928)$ & -4.778 & 0.0000 & $-1.1738(0.3122)$ & -3.760 & 0.0001 & $-3.7683(1.8830)$ & -2.001 & 0.0454 \\
\hline Sex & $0.0438(0.0850)$ & 0.516 & 0.6060 & $0.0004(0.0900)$ & 0.005 & 0.9963 & $0.0152(0.1581)$ & 0.096 & 0.9231 \\
\hline Age & $1.5964(1.5490)$ & 1.031 & 0.3027 & $3.9598(1.5979)$ & 2.478 & 0.0132 & $4.4780(2.9247)$ & 1.531 & 0.1257 \\
\hline Agesq & $-1.4679(1.6609)$ & -0.884 & 0.3768 & $-4.2481(1.6976)$ & -2.502 & 0.0123 & $-4.7754(3.1730)$ & -1.505 & 0.1323 \\
\hline Income & $-0.1727(0.1328)$ & -1.301 & 0.1933 & $-0.5177(0.1529)$ & -3.385 & 0.0071 & $-0.4759(0.2473)$ & -1.924 & 0.0544 \\
\hline Levyplus & $-0.1102(0.1138)$ & -0.969 & 0.3327 & $-0.1524(0.1192)$ & -1.279 & 0.2010 & $-0.3018(0.2088)$ & -1.446 & 0.1483 \\
\hline Freepoor & $-0.5447(0.2705)$ & -2.013 & 0.0441 & $0.0350(0.2643)$ & 0.133 & 0.8945 & $0.0458(0.5180)$ & 0.089 & 0.9295 \\
\hline Freerepa & $-0.1412(0.1428)$ & -0.989 & 0.3227 & $-0.4389(0.1458)$ & -3.008 & 0.0026 & $-0.4954(0.2683)$ & -1.846 & 0.0649 \\
\hline Illness & $0.0591(0.0292)$ & 2.021 & 0.0432 & $0.0787(0.0298)$ & 2.639 & 0.0083 & $0.0795(0.0550)$ & 1.447 & 0.1479 \\
\hline Actdays & $0.1161(0.0081)$ & 14.321 & 0.0000 & $0.1143(0.0071)$ & 16.091 & 0.0000 & $0.1574(0.0164)$ & 9.547 & 0.0000 \\
\hline Hscore & $0.0270(0.1458)$ & 1.852 & 0.0640 & $0.0045(0.0146)$ & 0.310 & 0.7566 & $0.0068(0.0285)$ & 0.240 & 0.8101 \\
\hline Chcond1 & $-0.0158(0.1063)$ & -0.149 & 0.8818 & $0.0237(0.1160)$ & 0.204 & 0.8382 & $0.0185(0.1893)$ & 0.098 & 0.9219 \\
\hline Chcond2 & $0.0958(0.1224)$ & 0.789 & 0.4339 & $-0.0001(0.1309)$ & -0.001 & 0.9992 & $0.1528(0.2296)$ & 0.666 & 0.5057 \\
\hline $\log (\beta)$ & & & & & & & $-2.5198(1.9617)$ & -1.284 & 0.1990 \\
\hline Zero-inflatic & n model coefficients & nomia & ogit link & & Zero-Hu & rdle mo & coefficients (bing & mial with & logit link): \\
\hline (Intercept) & $0.5707(0.8758)$ & 0.652 & 0.5146 & $-2.2899(0.2772)$ & -8.259 & 0.0000 & $-2.2899(0.2772)$ & -8.259 & 0.0000 \\
\hline Sex & $-0.6757(0.2676)$ & -2.525 & 0.0115 & $0.2606(0.0823)$ & 3.166 & 0.0015 & $0.2606(0.0823)$ & 3.166 & 0.0015 \\
\hline Age & $11.0850(5.2032)$ & 2.130 & 0.0331 & $-1.9760(1.5271)$ & -1.294 & 0.1956 & $-1.9760(1.5271)$ & -1.294 & 0.1956 \\
\hline Agesq & $-14.1677(5.9656)$ & -2.375 & 0.1755 & $2.7366(1.6806)$ & 1.628 & 0.1034 & $2.7366(1.6806)$ & 1.628 & 0.1034 \\
\hline Income & $-0.3300(0.3991)$ & -0.827 & 0.4083 & $0.0074(0.1273)$ & 0.059 & 0.9533 & $0.0074(0.1273)$ & 0.059 & 0.9533 \\
\hline Levyplus & $-0.8634(0.3113)$ & -2.773 & 0.0055 & $0.2670(0.1006)$ & 2.654 & 0.0079 & $0.2670(0.1006)$ & 2.654 & 0.0079 \\
\hline Freepoor & $-0.1122(0.7263)$ & -0.155 & 0.8771 & $-0.6803(0.2610)$ & -2.606 & 0.0091 & $-0.6803(0.2610)$ & -2.606 & 0.0091 \\
\hline Freerepa & $-1.4768(0.5452)$ & -2.708 & 0.0067 & $0.4162(0.1398)$ & 2.976 & 0.0029 & $0.4162(0.1398)$ & 2.976 & 0.0029 \\
\hline Illness & $-1.0110(0.2246)$ & -4.501 & 0.0000 & $0.2634(0.0289)$ & 9.096 & 0.0000 & $0.2634(0.0289)$ & 9.096 & 0.0000 \\
\hline Actdays & $-15.5567(1386.8329)$ & -0.011 & 0.9910 & $0.1580(0.0119)$ & 13.259 & 0.0000 & $0.1580(0.0119)$ & 13.259 & 0.0000 \\
\hline Hscore & $-0.1197(0.0756)$ & -1.583 & 0.1133 & $0.0634(0.0174)$ & 3.644 & 0.0002 & $0.0634(0.0174)$ & 3.644 & 0.0002 \\
\hline Chcond1 & $-0.2281(0.3319)$ & -0.687 & 0.4918 & $0.1020(0.0913)$ & 1.117 & 0.2641 & $0.1020(0.0913)$ & 1.117 & 0.2641 \\
\hline Chcond2 & $-0.3563(0.4948)$ & -0.720 & 0.4714 & $0.2667(0.1259)$ & 2.118 & 0.0341 & $0.2667(0.1259)$ & 2.118 & 0.0341 \\
\hline$\ell$ & -3117.022 & & & -3212.58 & & & -3144.939 & & \\
\hline $\mathrm{AIC}$ & 6286.043 & & & 6477.160 & & & 6343.877 & & \\
\hline $\mathrm{BIC}$ & 6456.460 & & & 6647.577 & & & 6520.848 & & \\
\hline
\end{tabular}

Zero-inf. CosPois reg. model

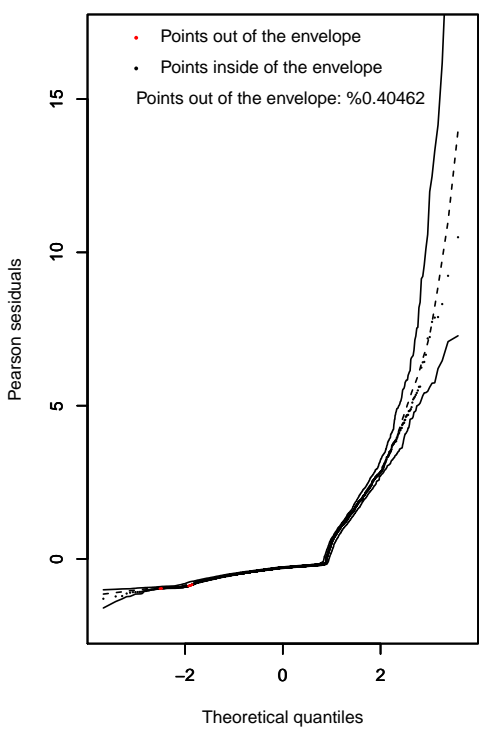

Zero-inf. Poisson reg. model

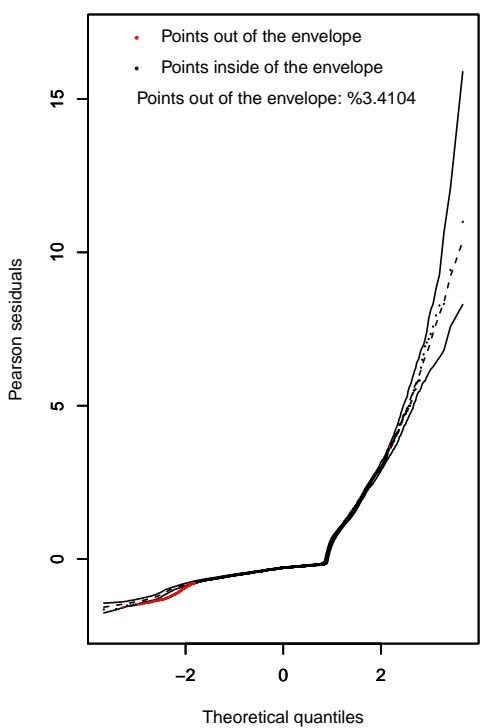

Zero-inf. neg-bin reg. model

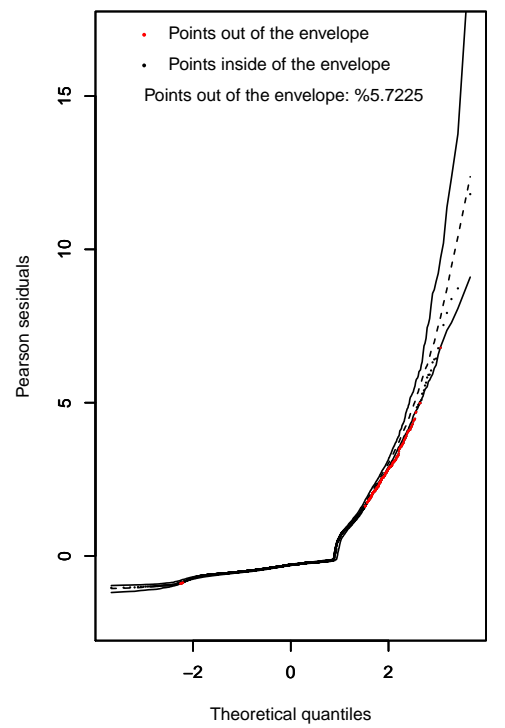

Figure 6. QQ-plots and simulated envelopes for the Pearson residuals 


\section{References}

[1] Y. Akdoğan, C. Kuş, A. Asgharzadeh, İ. Kınacı and F. Sharafi, Uniform-geometric distribution, J. Stat. Comput. Simul. 86 (9), 1754-1774, 2016.

[2] A. Atkinson, Two graphical displays for outlying and influential observations in regression, Biometrika 68 (1), 13-20, 1981.

[3] H.S. Bakouch, A weighted negative binomial Lindley distribution with applications to dispersed data, An. Acad. Brasil. Ciênc. 90 (3), 2617-2642, 2018.

[4] D. Bhati and S. Joshi, Weighted geometric distribution with a new characterization of geometric distribution, Comm. Statist. Theory Methods 47 (6), 1510-1527, 2018.

[5] A.C. Cameron, P.K. Trivedi, F. Milne and J. Piggott, A microeconomic model of the demand for health care and health insurance in Australia, Rev. Econ. Stud. 55 (1), 85-106, 1988.

[6] J.D. Castillo and M.P. Casany, Weighted Poisson Distributions and under dispersion Situations, Ann. Inst. Statist. Math. 5, 567-585, 1998.

[7] C. Chesneau, H.S. Bakouch, T. Hussain and B.A. Para, The cosine geometric distribution with count data modeling, J. Appl. Stat. 48 (1), 124-137, 2021.

[8] D.A.S. Fraser, Probability and Statistics: Theory and Applications, North Scituate MA, Duxbury Press, 1976.

[9] M.G. Kendall, Natural law in social sciences, J. Roy. Statist. Soc. Ser. A 124, 1-19, 1961.

[10] M.S.A. Khan, A. Khalique and A.M. Abouammoh, On estimating parameters in a discrete, Weibull distribution, IEEE Trans. Reliab. 38 (3), 348-350, 1989.

[11] C. Kuş, Y. Akdoğan, A. Asgharzadeh, İ. Kınacı and K. Karakaya, Binomial-discrete Lindley distribution, Commun. Fac. Sci. Univ. Ank. Ser. A1. Math. Stat. 68 (1), 401-411, 2018.

[12] A.J. Lemonte, G. Moreno-Arenas and F. Castellares, Zero inflated Bell regression models for count data, J. Appl. Stat. 47 (2), 265-286, 2020.

[13] G.P. Patil and C.R. Rao, The weighted distributions: A survey and their applications, in: Krishnaiah, P.R. (ed.) Applications of statistics, North-Holland Publ. Co., Amsterdam, 1977.

[14] G.P. Patil and C.R. Rao, Weighted distributions and size biased sampling with applications to wild-life populations and human families, Biometrica 34, 179-189, 1978.

[15] C.R. Rao, On discrete distributions arising out of methods of ascertainment, Sankhya A, 311-324, 1965. 